\title{
Superradiant and stimulated-superradiant emission in prebunched electron-beam radiators. II. Radiation enhancement schemes
}

\author{
A. Gover and E. Dyunin \\ Faculty of Engineering, Department of Physical Electronics, Tel-Aviv University, Tel-Aviv 69978 Israel \\ Y. Lurie, Y. Pinhasi, and M. V. Krongauz \\ Faculty of Engineering, Department of Electrical and Electronic Engineering, The College of Judea and Samaria, Ariel 44837 Israel \\ (Received 1 March 2004; published 2 March 2005)
}

\begin{abstract}
Further enhancement of the intense coherent superradiant and stimulated-superradiant emission from prebunched electron beams is possible, in schemes of prebunched beam radiation devices, and particularly free electron laser (FEL). The enhancement of coherent power and spectral power by use of a waveguide, particularly at the zero-slippage condition, is evaluated. A special scheme of a stimulated-superradiance FEL oscillator is analyzed and is shown to feature ultimate radiative energy conversion efficiency (near $100 \%)$.
\end{abstract}

DOI: $10.1103 /$ PhysRevSTAB.8.030702

PACS numbers: 41.60.Cr, 52.59.-f, 42.50.Fx

\section{INTRODUCTION}

In a companion article (part I, Ref. [1]), the formulation of superradiant (SR) and stimulated-superradiant (ST-SR) radiative emission processes in prebunched electron-beam coherent radiation sources was derived. Special attention was given to the understanding and comparison between the prebunched beam (PB-FEL) [2-14] and the coherent synchrotron radiation (CSR) radiation sources [15].

The main results of the formulation are general expressions for the characteristic optical parameters of radiation emitted by the two different coherent emission processes of SR and ST-SR emission. Choosing to characterize the optical radiation in terms of discrete transverse (free space or waveguide) radiation modes, the coherence optical properties of SR and ST-SR emission are described in terms of the spectral energy emission per mode. The generic expressions of these parameters for any $e$-beam radiation scheme are

$$
\begin{aligned}
& \left(\frac{d W_{q}}{d \omega}\right)_{\mathrm{SR}}=\frac{N^{2}}{8 \pi \mathrm{P}_{q}}\left|\Delta \mathrm{W}_{q e}^{(0)}(\omega)\right|^{2}\left|M_{b}(\omega)\right|^{2}\left|M_{M}(\omega)\right|^{2}, \\
& \left(\frac{d W_{q}}{d \omega}\right)_{\mathrm{ST}-\mathrm{SR}}=\frac{N}{2 \pi}\left|C_{q}^{\mathrm{in}}(\omega)\right|\left|\Delta \mathrm{W}_{q e}^{(0)}(\omega)\right|\left|M_{b}(\omega)\right| \\
& \times\left|M_{M}(\omega)\right| \cos \varphi,
\end{aligned}
$$

where $\mathrm{P}_{q}$ is the (arbitrary) normalization power of the relevant mode $q,\left|C_{q}^{\text {in }}(\omega)\right|$ and $\varphi$ are, respectively, the amplitude of the spectral Fourier component of the radiation mode, inserted into the interaction region (in the case of ST-SR), and the phase of the electron bunch relative to this radiation mode. $N$ is the total number of electrons in the bunched beam that may be composed of a macropulse of $N_{M}$ microbunches, each having $N_{b}$ electrons per bunch $\left(N=N_{M} N_{b}\right)$. The microbunch and macropulse form factors $M_{b}(\omega), M_{M}(\omega)$ are the spectral Fourier components of the microbunch and macropulse current waveforms. For a uniform macropulse, composed of electron bunches of equal currents $-e N_{b} f\left(t_{0}^{\prime}-t_{0 k}\right)\left(k=1, \ldots, N_{M}\right)$ streaming at bunch repetition rate $f_{b}=\omega_{b} / 2 \pi\left[f\left(t_{0}^{\prime}\right)\right.$ is normalized according to $\left.\int_{t_{0 k}-\pi / \omega_{b}}^{t_{0 k}+\pi / \omega_{b}} f\left(t_{0}^{\prime}\right) d t_{0}^{\prime}=1\right]$,

$$
\begin{gathered}
M_{b}(\omega)=\int_{-\pi / \omega_{b}}^{\pi / \omega_{b}} f\left(t_{0}^{\prime}\right) e^{i \omega t_{0}^{\prime}} d t_{0}^{\prime}, \\
M_{M}(\omega)=\frac{\sin \left(N_{M} \pi \omega / \omega_{b}\right)}{N_{M} \sin \left(\pi \omega / \omega_{b}\right)} .
\end{gathered}
$$

The "complex work function" of a single electron $\Delta \mathrm{W}_{q e}(\omega)$

$$
\Delta \mathrm{W}_{q e}^{(0)}=-e \int_{-\infty}^{\infty} v_{e}^{(0)}(t) \cdot \tilde{\mathbf{E}}_{q}^{*}\left(\mathbf{r}_{e}^{(0)}(t)\right) e^{i \omega t} d t
$$

is the only factor in (1) and (2) that depends on the particular radiation scheme and can be evaluated for the given radiation mode $\tilde{\mathbf{E}}_{q}(\mathbf{r})=\mathbf{E}_{q}\left(\mathbf{r}_{\perp}\right) e^{i k_{z q} z}$ if the electron trajectories along the interaction region $\left\{\mathbf{r}_{e}^{(0)}(t), \mathbf{v}_{e}^{(0)}(t)\right\}$ are known.

Based on these results, and concentrating now on the radiation scheme of PB-FEL (extension to other kinds of radiation schemes is possible), we will explore in this paper how the SR emission can be enhanced (mainly by employing a waveguide structure) and how the concept of ST-SR emission in PB-FEL can be extended to the nonlinear (saturation) regime and employed in an oscillator configuration of a unique property of ultimate efficiency of radiative energy conversion. 


\section{ENHANCEMENT OF SUPERRADIANT EMISSION IN PREBUNCHED ELECTRON-BEAM RADIATORS}

Examination of the generic formulas (1) and (5) reveals that, for all radiation schemes, the spectral work function grows in proportion to the interaction time $t_{r}$ (or interaction length $L$ ), if the electron oscillation can keep in phase with a radiation mode along the interaction length. Consequently, the spectral emission energy grows, in general, in proportion to $L^{2}$ : in PB-FEL, proportionally to the number of undulator periods $\left(N_{w}\right)$ squared; in periodic transition radiation [16], proportionally to the number of plates squared; in Cerenkov radiation, proportionally to the length of the dielectric material $L$ squared, and so on.

For the specific example of PB-FEL, the spectral energy emission per mode (1) was found to be

$$
\begin{aligned}
\left(\frac{d W_{q}}{d \omega}\right)_{\mathrm{SR}}= & \frac{N^{2} e^{2} Z_{q}}{16 \pi} \cdot \frac{\left|\mathbf{v}_{\perp 0} \cdot \hat{\mathbf{e}}_{q}^{*}\right|^{2}}{\boldsymbol{v}_{z}^{2}} \cdot \frac{L^{2}}{A_{\mathrm{em} q}} \\
& \times \sin c^{2}(\theta L / 2)\left|M_{b}(\omega)\right|^{2}\left|M_{M}(\omega)\right|^{2},
\end{aligned}
$$

where

$$
\theta(\omega)=\frac{\omega}{v_{z}}-k_{z q}(\omega)-k_{w}
$$

is the detuning parameter, $k_{z q}(\omega)$ is the axial wave number of mode $q, k_{w}=2 \pi / \lambda_{w}$ is the wiggler wave number, $\mathbf{v}_{\perp 0}$ is the transverse wiggler velocity, $\hat{e}_{q}=\mathrm{E}_{q}(0,0) /\left|\mathrm{E}_{q}(0,0)\right|$ is a unit vector in the direction of the field of the radiation mode $q, Z_{q}$ is the mode impedance (in free space and for the fundamental mode of an overmoded waveguide $Z_{q}=$ $\sqrt{\mu / \varepsilon}$ ), and the effective mode area is defined by

$$
A_{\mathrm{em} q}=\frac{\mathrm{P}_{q}}{\left|\mathrm{E}_{q \perp}(0,0)\right|^{2} /\left(2 Z_{q}\right)} .
$$

This expression (6) exhibits the $L^{2}$ dependence of the spectral energy on $L$. However, the scaling of other radiation parameters, such as the total energy emission into a mode [the frequency integral of (6)], may be different.

In free-space interaction, especially at long wavelengths, the effective interaction length may be limited by diffraction. The assumption of interaction with a uniform radiation field along the interaction length $L$ is only valid for about two Rayleigh lengths,

$$
L \cong 2 z_{R}=2 \pi w_{0}^{2} / \lambda,
$$

where we assume interaction with a fundamental Gaussian mode [17] having its waist of size $w_{0}$ at the undulator center [see Fig. 1(a)]. Alternatively stated, for a given interaction length $L$, the free-space radiation mode of choice (determined by the radiation collection optics), that will carry the highest coherent energy emitted from the beam, has cross section dimensions with mode effective area (8) [18]

$$
A_{\mathrm{em}}=\pi w_{0}^{2} / 2=\lambda L / 4,
$$

which reduces (6) into

$$
\begin{aligned}
\left(\frac{d W_{q}}{d \omega}\right)_{\mathrm{SR}}= & \frac{N_{b}^{2} e^{2}}{4 \pi} \cdot \sqrt{\frac{\mu}{\varepsilon}} \cdot \frac{\left|\mathbf{v}_{\perp 0} \cdot \hat{\mathbf{e}}_{q}^{*}\right|^{2}}{v_{z}^{2}} \cdot \frac{L}{\lambda} \\
& \times \sin c^{2}(\theta L / 2)\left|M_{b}(\omega)\right|^{2}\left|M_{M}(\omega)\right|^{2} .
\end{aligned}
$$

We observe that the scaling with $L$ of the spectral energy per mode in free-space interaction is linear (11). However, evaluate now the total energy per mode emitted by a single short bunch $\left(\left|M_{b}(\omega)\right|=\left|M_{M}(\omega)\right|=1\right)$ by integrating (11) over all frequencies $\omega$. Consider ones that in free space $\left(k_{z q}=\omega / c\right)$ the peak (synchronism) frequency and bandwidth of the spectral function $\sin c^{2}(\theta L / 2)$ are

$$
\begin{gathered}
\omega_{0}=\frac{2 \gamma^{2}}{1+a_{w}^{2} / 2} c k_{w}, \\
\Delta \omega=\frac{\omega_{0}}{N_{w}},
\end{gathered}
$$

where $a_{w}=\gamma v_{\perp 0} / c$ is the wiggler parameter. This results in the expression for the total emission energy per mode ${ }^{1}$ :

$$
\begin{aligned}
\left(W_{q}\right)_{\mathrm{SR}} & =\frac{N_{b}^{2} e^{2}}{2 \varepsilon} \cdot \frac{\left|\tilde{\mathbf{v}}_{\perp 0} \cdot \hat{\mathbf{e}}_{q}^{*}\right|^{2}}{\boldsymbol{v}_{z 0}^{2}} \cdot \frac{\lambda_{w}}{\lambda^{2}} \\
& \cong \frac{2}{\pi} \cdot \sqrt{\frac{\mu}{\varepsilon}} N_{b}^{2} e^{2} \omega_{0} \cdot \frac{a_{w}^{2}}{1+a_{w}^{2} / 2} A_{J J}^{2} .
\end{aligned}
$$

This parameter, interestingly enough, is entirely independent of $L$ (consistent with the result of [5]).

A more complete characterization of the radiation beam emitted from the bunched $e$-beam can be made in terms of the Wigner distribution of the transverse space-angle phase space, which is related to the brightness field of the radiation source $[19,20]$. This was calculated for a narrow $e$-beam, radiating in free space in a long undulator, by using a plane-wave expansion instead of a discrete mode expansion, resulting in the phase-space distribution shown in Fig. 2 [21]. This distribution has a volume in $x-\theta_{x}$ space of $\lambda / 2$, similar to the phase-space volume of a Gaussian wave. However, its irregular shape does not permit efficient matching into a useful spatially coherent radiation mode, and some radiation energy may be lost when the radiated wave will be collected by apertured optics into a spatially coherent phase-space volume. The source of this detrimental feature is illustrated schematically in Fig. 1(b), which displays diffracting radiation wavelets, emitted from a

\footnotetext{
${ }^{1}$ In the second part of (14) we assumed specifically a linearly polarized wiggler, and added the wiggler-harmonics Besselfunction factor [19] $A_{J J}=\left[J_{0}\left(a_{w}^{2} /\left(4+2 a_{w}^{2}\right)\right)-J_{1}\left(a_{w}^{2} /(4+\right.\right.$ $\left.\left.\left.2 a_{w}^{2}\right)\right)\right]$. This factor needs to be included only in the case of a strong wiggler parameter $\left(a_{w} \gg 1\right)$. In the case of a helical wiggler [18], one should substitute $A_{J J}=1$, and in the denominator of (14) and in (12) $a_{w}^{2} / 2 \rightarrow a_{w}^{2}$.
} 
a)

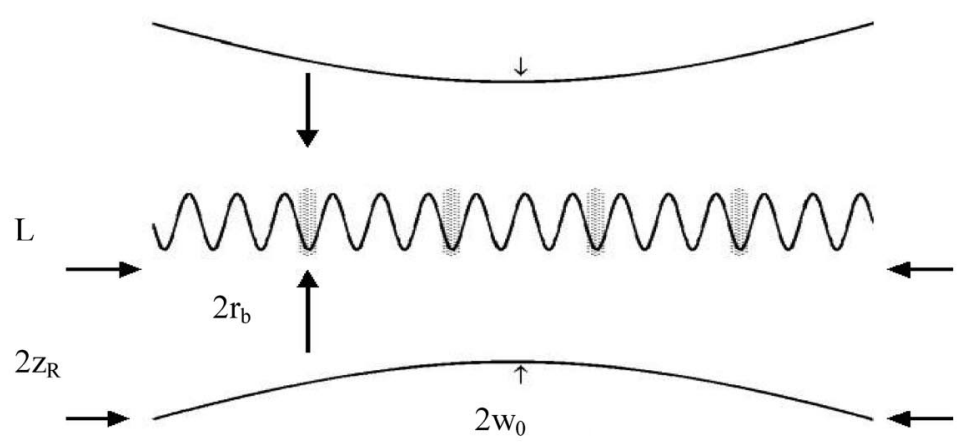

b)

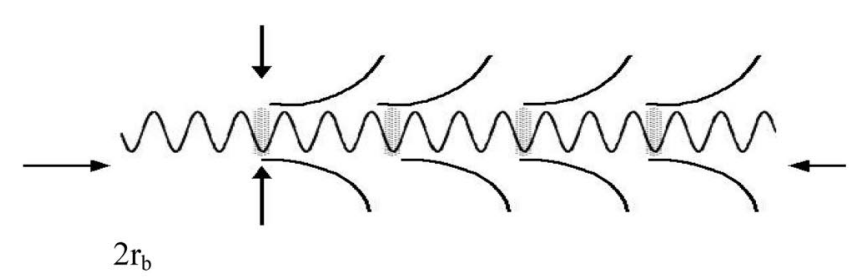

c)

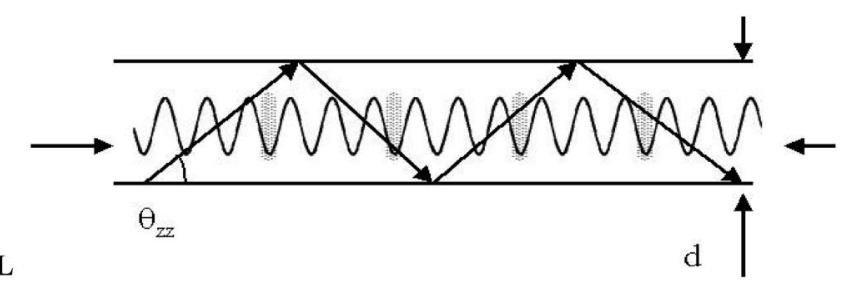

FIG. 1. Superradiant emission of an undulating, finite width, bunched $e$-beam: (a) emission into a free-space radiation mode (Gaussian wave) of waist size $w_{0}$, (b) diffraction-dominated emission of wavelets in free space in a long wiggler, and (c) emission into a single waveguide mode characterized by ray zigzag angle $\theta_{Z Z}$.

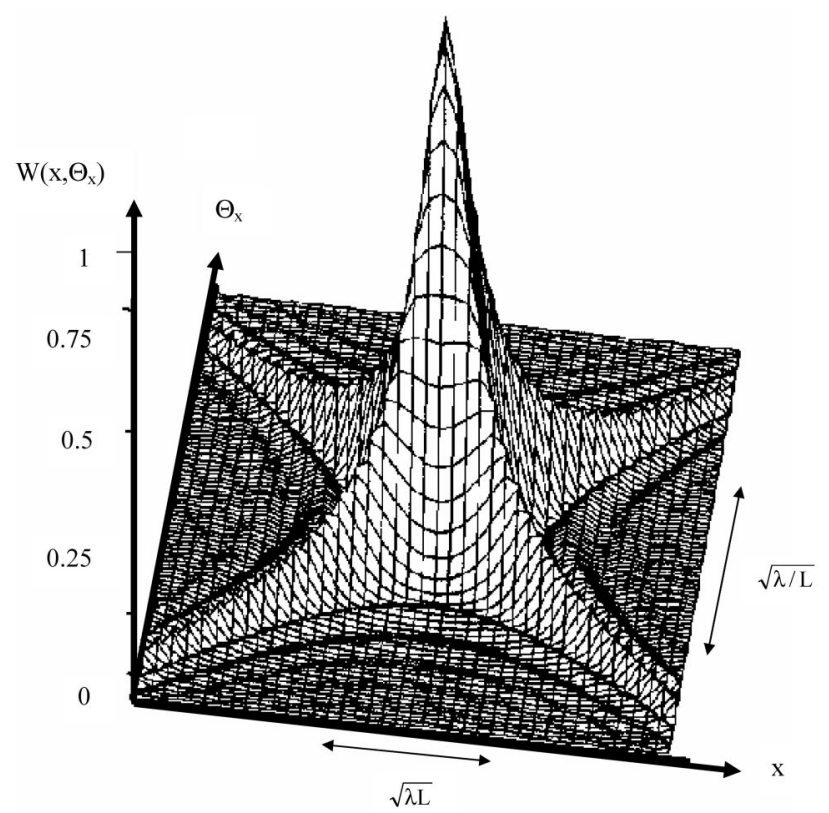

FIG. 2. The Wigner distribution function of undulatorsynchrotron radiation in $2 \mathrm{D}$ space-angle $\left(X, \Theta_{x}\right)$ phase space [21]. finite width electron bunch at different points along the $e$-beam propagation path. This emitted radiation pattern is spread out in phase space in a less regular way than the Gaussian radiation mode [Fig. 1(a)], which has a Gaussian Wigner distribution.

\section{A. Radiation enhancement in a waveguide}

At long wavelengths the optical diffraction effect inhibits efficient interaction between the electron beam and a free-space radiation mode along a long path. For a finite $e$-beam radius $r_{b}$, the free-space radiation mode crosssection area, $A_{\mathrm{em}}=\pi \omega_{0}^{2} / 2=\lambda L / 4$ (10), becomes larger than the $e$-beam cross section $\pi r_{b}^{2}$ whenever the interaction length $L$ exceeds twice the "Rayleigh length" associated with the $e$-beam width:

$$
L>2 \pi r_{b}^{2} / \lambda .
$$

When this happens, the effective mode area $A_{\mathrm{em}}(10)$ starts growing with $L$, and therefore the emitted radiation spectral energy scales then in proportion to $L$ (11) instead of the quadratic growth in (6). 
Clearly, the diffraction effect is more detrimental at long wavelengths. This is definitely a practical concern with superradiant emission from bunched electrons, because the present state of the art of $e$-beam bunching and the tight bunching condition $t_{b} \ll 2 \pi / \omega$ still limit superradiant emission to $\mathrm{THz}$ frequencies, which correspond to quite long optical wavelengths. Consider, for example, emission at $1 \mathrm{THz}$ frequency $(\lambda=300 \mu \mathrm{m})$ from an electron beam of radius $r_{b}=1 \mathrm{~mm}$. Condition (15) is satisfied then already for $L>20 \mathrm{~mm}$, and therefore in all practical situations of free-space emission, extending the interaction length $L$ increases the radiation spectral energy only proportionately according to (11), and the total emitted coherent radiation energy (14) does not grow at all with $L$.

A practical way to overcome the detrimental effect of diffraction at long wavelengths is the use of a waveguide, as shown in Fig. 1(c). At long wavelengths the effective cross-section area $A_{\mathrm{em} q}(8)$ corresponding to the waveguide cross-sectional dimensions (limited primarily by the width of the $e$-beam) is smaller than the free-space propagation effective mode area (10). Then using a waveguide enhances the radiative emission. The radiation spectral energy then scales quadratically with $L$ (6), even for long wiggler length $L$, and the total coherent radiation emission energy $W_{q}$ still grows (in proportion) with $L$.

Overmoded standard waveguides of circular or rectangular cross section may be considered for use in this scheme, as well as specialized waveguides, such as (curved) parallel plates, metal-dielectric and corrugated waveguides, which are designed to attain low Ohmic absorption losses on the walls and small mode cross-section area $A_{\mathrm{em} q}[18,22]$. Assuming $Z_{q} \cong \sqrt{\mu / \varepsilon}$ also for the overmoded waveguide, the enhancement factor of the waveguide scheme relative to the free-space propagation scheme [ratio of (6) to (11)] is

$$
\frac{\text { waveguide } \mathrm{SR}}{\text { free-space } \mathrm{SR}}=\frac{\lambda L / 4}{A_{\mathrm{em} q}} .
$$

An interesting physical interpretation of the waveguide enhancement factor can be derived from the "zigzag" ray picture of a waveguide radiation mode depicted in Fig. 1(c) (also used in describing waveguide modes in other $e$-beam radiation devices like gyrotrons [23]). It can be argued that the "plane-wave" wavelets, emitted by the electron bunch along the waveguide, are reflected by the walls, and add up coherently at the end of the waveguide. The radiation is then enhanced by a factor

$$
N_{z z}=\frac{L}{d / \sin \theta_{z z}}=\frac{L}{d} \frac{k_{\perp}}{k}
$$

which is the number of zigzag ray reflections $\left(\theta_{Z Z}\right.$, the mode zigzag angle; $d$, the waveguide width; $k_{\perp q}=$ $\omega_{\text {c.o.q. }} / c$, the mode transverse wave number; $k=2 \pi / \lambda$ ). If we take, for example, the $\mathrm{TE}_{01}$ mode of a square rect- angular waveguide of cross-sectional dimension $d$, its effective cross section area [18] is $A_{\text {eff }}=d^{2} / 2$, and $k_{\perp}=\pi / d$. The enhancement factor (16) is found in this case to be identical to the number of zigzag reflections: $N_{z z}=(1 / 2) \cdot \lambda L / d^{2}$. In another practical example-the fundamental linearly polarized hybrid mode of a (corrugated) circular cross-section waveguide of diameter $d$, $\left|\mathbf{E}_{q x}(\mathbf{r})\right|=J_{0}(2 u r / d)$ (where $u=2.405$ is the first zero of the zero order Bessel function), the effective crosssection area is [18] $A_{\mathrm{em} q}=0.27 \pi(d / 2)^{2}$ and $k_{\perp}=2 u / d$. The enhancement factor (16) turns out to be, in this case, $4 \lambda L / \pi d^{2}$, and the number of zigzag reflections, $N_{z z}=$ $2.4 \lambda L / \pi d^{2}$, is quite similar.

It is interesting to apply this formulation to an example of a real experiment design. Consider the far-infraredvacuum ultraviolet pump-probe facility design proposed by Faatz et al. [5] based on the TESLA test facility at DESY. In this design $E \approx 1 \mathrm{GeV}, \lambda_{w}=0.6 \mathrm{~m}, N_{w}=10$, $B_{w} \approx 1.2 \mathrm{~T}\left(a_{w}=67.3\right), e N_{e}=1 \mathrm{nC}$. Equation (14) predicts coherent superradiant emission of coherent radiation energy of $W_{q}=0.8 \mathrm{~mJ}$ per microbunch at frequency $1 \mathrm{THz}(\lambda=300 \mu \mathrm{m})$. If a (rectangular) waveguide of cross-section dimension $d=5 \mathrm{~mm}$ is used, the enhancement factor (16) is $\times 36$, and the expected coherent superradiant energy will be $W_{q}=29 \mathrm{~mJ}$.

\section{B. Ultrashort radiation pulses at zero-slippage conditions}

A special feature of superradiant emission in a waveguide that can be taken advantage of for enhancement of the radiation parameters is the possibility to attain ultrashort wide bandwidth radiation pulses at the zero-slippage ("grazing") condition [4].

The sinc function in the expression for the radiation spectral energy of the PB-FEL (6) attains its maximum value at the synchronism frequency condition

$$
\theta(\omega)=\omega / v_{z}-k_{z q}(\omega)-k_{w}=0 .
$$

Figure 3 depicts the graphical solution of this equation as the intersection between the beam line $\omega / v_{z}-k_{w}$ and the waveguide mode dispersion relation:

$$
k_{z q}(\omega)=\left(\omega^{2}-\omega_{\text {c.o.q. }}^{2}\right)^{1 / 2} / c .
$$

One can see that usually the equation (18) has two solutions [24] (corresponding to forward and backward emission in the electron beam reference frame). At the high frequency solution the radiation group velocity $v_{g q}=$ $d \omega / d k_{z}$ is larger than the electron velocity $v_{z}$, and vice versa at the lower frequency solution. Thus, the wave packet emitted by the electron bunch is composed of two wave packets: a fast high frequency one, slipping ahead of the electron bunch, and a low frequency one, trailing behind (see Fig. 4). 


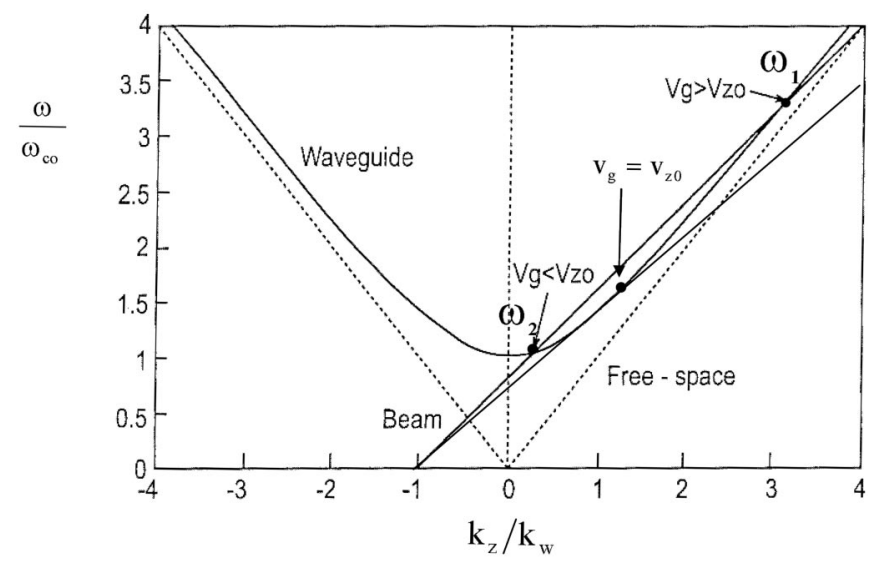

FIG. 3. The crossing of the waveguide mode and beam line dispersion curves in the cases of two frequencies and zeroslippage solutions.

In order to find out the time domain waveform of the radiation wave packet emitted by the electron bunch, one must resort to the complex frequency-domain expression of this field (derived in part I),

$$
\begin{gathered}
\mathbf{E}(\mathbf{r}, \omega)=\sum_{q} C_{q}(L, \omega) \mathbf{E}_{q}(x, y) e^{i k_{z q}(\omega) L}, \\
C_{q}(L, \omega)=-\frac{1}{4 P_{q}} \sum_{j=1}^{N} \Delta \mathbf{W}_{q j}^{0}, \\
\Delta \mathbf{W}_{q j}^{0}=-e \frac{\mathbf{v}_{\perp 0} \cdot \mathbf{E}_{q}^{*}}{2 v_{z}} L \sin c\left(\theta_{q} L / 2\right) e^{i \theta_{q} L / 2} e^{i \omega t_{o j},}
\end{gathered}
$$

and employ the inverse Fourier transform. This can be performed numerically for any electron beam distribution and bunching parameters. In certain limits the transform can be carried out analytically.

Consider superradiant emission from a PB-FEL with a tightly bunched electron beam $\left(t_{o j}=0\right.$ for all electrons or alternatively stated: $\left.M_{b}=M_{M}=1\right)$. The frequency dependence of the field (20) is expressed predominantly

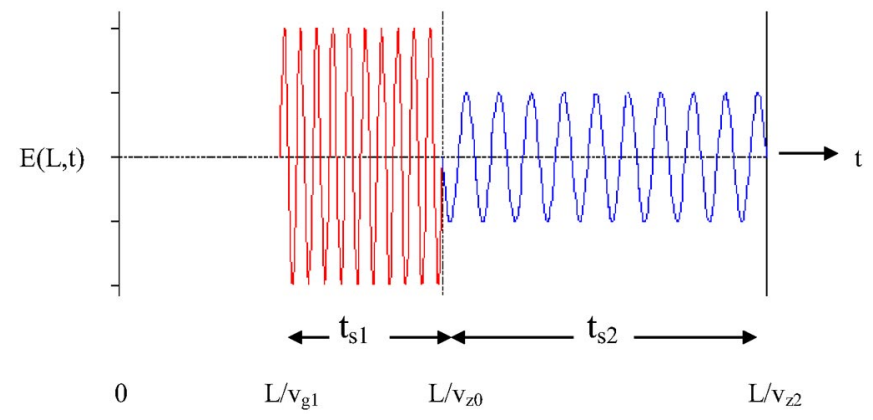

FIG. 4. (Color) The temporal waveform dependence of the field of a radiation wave packet emitted in free space in the forward direction from an $e$-beam bunch entering the wiggler $(z=0)$ at $t=0$, and observed at the wiggler exit $(z=L)$. through the parameters $k_{z q}(\omega)(19)$ and $\theta_{q}(\omega)(7)$. When the two solutions of Eq. (18) $\left(\omega_{1}, \omega_{2}\right)$ (see Fig. 3) are well spaced apart, the detuning parameter $\theta(\omega)$ can be expanded to first order around the center frequencies $\omega_{1}, \omega_{2}$ :

$$
\theta(\omega) \cong\left(\omega-\omega_{1,2}\right) t_{s 1,2},
$$

where

$$
\begin{gathered}
t_{s 1,2}=\frac{L}{v_{z}}-\frac{L}{v_{g 1,2}}, \\
v_{g 1,2}=\left.\frac{d \omega}{d k_{z}}\right|_{\omega 1,2}
\end{gathered}
$$

are the slippage times and group velocities of the wave packets $\omega_{1}$ and $\omega_{2}$.

In this case the inverse Fourier transform of (20) can be carried out analytically [4], resulting in two uniform wave packets extending ahead and behind the electron bunch with slippage times $t_{s 1}$ and $t_{s 2}$, respectively (see Fig. 4). The frequency bandwidths of the radiation wave packets, determined by the width of the $\sin c(\theta L)$ function $[\Delta \theta(\omega) L=2 \pi]$ is then

$$
\Delta f_{1,2}=\frac{\Delta \omega_{1,2}}{2 \pi}=\frac{1}{t_{s 1,2}}=\frac{c / L}{\beta_{z}^{-1}-\beta_{g 1,2}^{-1}},
$$

which is exactly the Fourier transform limit of the wave packet pulse durations.

In free-space emission, or when the waveguide dispersion effect is negligible $\left(\omega_{c 0} \ll \omega\right)$, there is only a single solution to Eq. (18) that corresponds to a forward propagating wave. In this case $v_{g 1}=c$, and the slippage time and corresponding frequency bandwidth of the radiation wave packet are

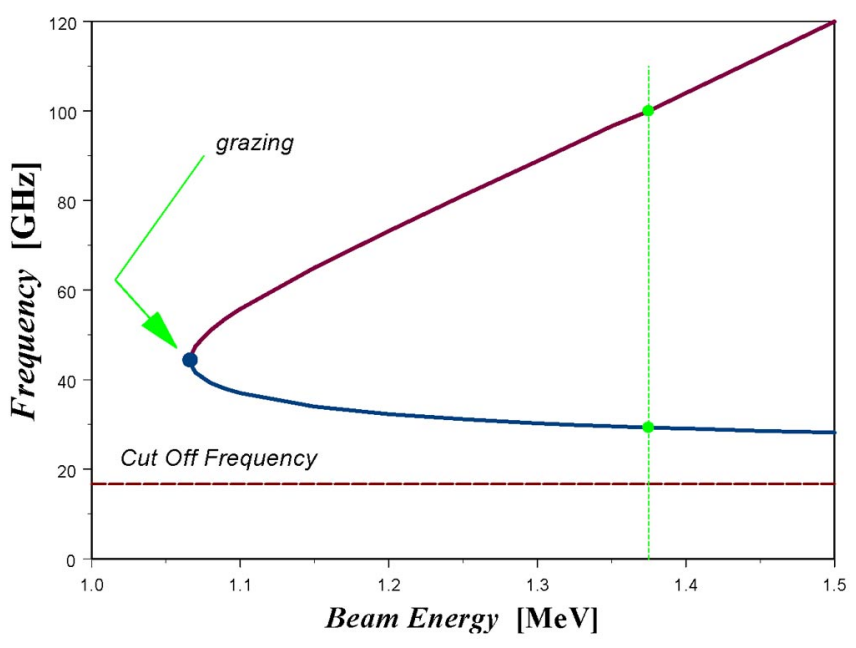

FIG. 5. (Color) Dispersive waveguide solutions for the radiation frequencies as a function of the acceleration beam energy. 


$$
\begin{gathered}
t_{s}=\frac{L}{c}\left(\beta_{z}^{-1}-1\right)=\frac{N_{w}}{f_{0}}, \\
\Delta \omega=\frac{\omega_{0}}{N_{w}} .
\end{gathered}
$$

The pulse duration of the wave packet emitted in a dispersive waveguide (24) is shorter than in free space (27),

(a)

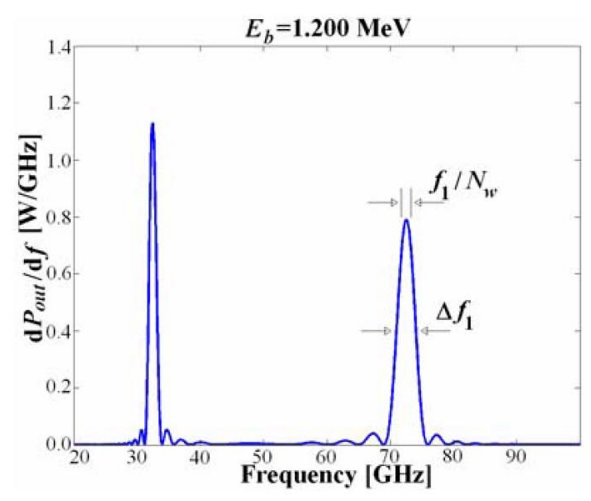

(c)

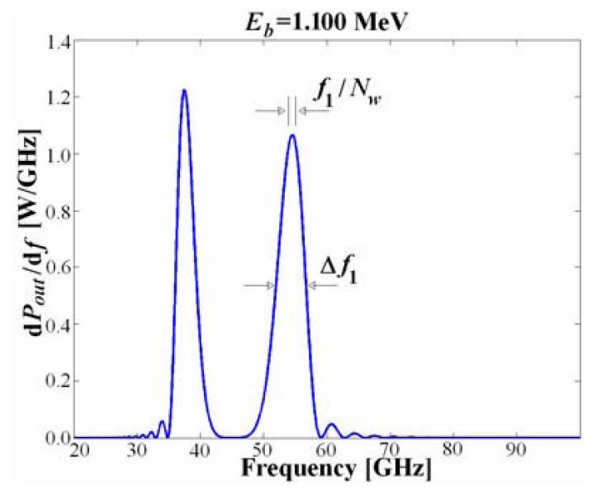

(e)

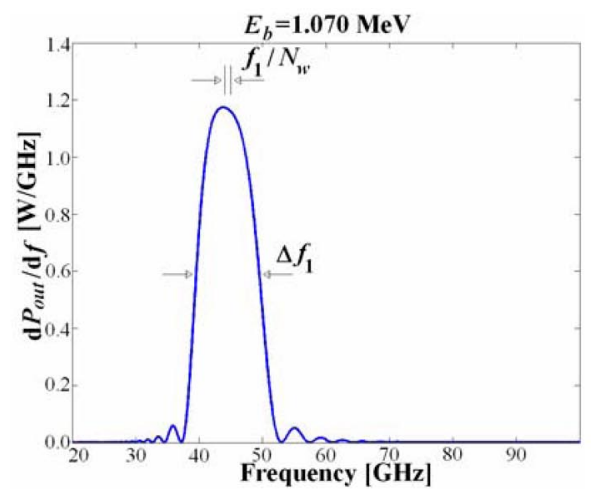

and correspondingly its frequency bandwidth is wider (26) and (28).

Inspection of Eq. (24) and Fig. 3 suggests a "zeroslippage" condition when the beam line $\omega / v_{z}-k_{w}$ and waveguide dispersion curve $\omega\left(k_{z}\right)$ are tangent ("grazing condition"):

$$
v_{g q}=v_{z} .
$$

In this limit one would expect a short duration of the

(b)

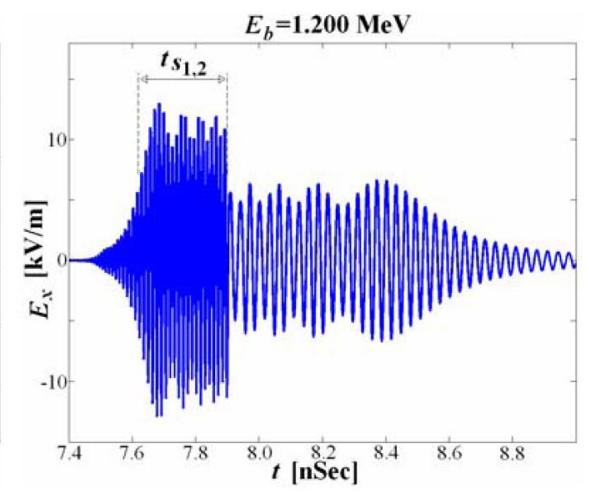

(d)

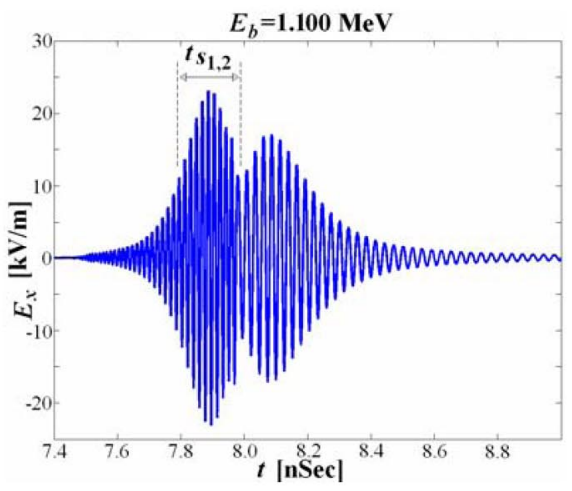

$(f)$

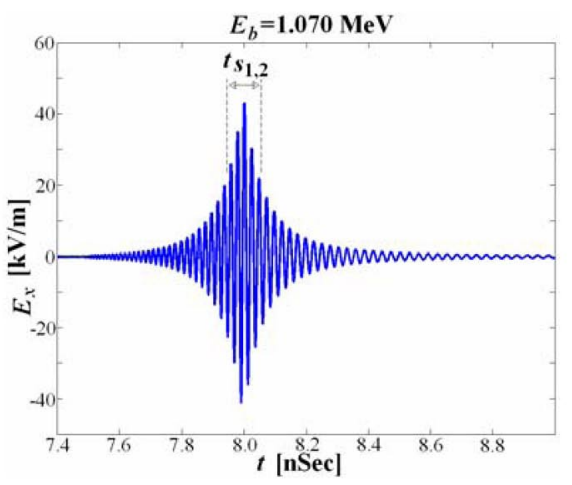

FIG. 6. (Color) The spectral power (a),(c),(e) and radiation field waveform (b),(d),(f) of a radiation wave packet emitted from a short $e$-beam bunch computed with an FEL code numerical simulation: (a),(b) $E_{k}=1200 \mathrm{keV}$, well spaced apart two frequencies solution; (c),(d) $E_{k}=1100 \mathrm{keV}$, near "grazing intersection"; (e),(f) $E_{k}=1070 \mathrm{keV}$, at "grazing intersection point." 
TABLE I. Pulse compression and bandwidth expansion in a dispersive waveguide.

\begin{tabular}{lccc}
\hline \hline \multicolumn{1}{c}{ Parameter } & Figs. 6(a) and 6(b) & Figs. 6(c) and 6(d) & Figs. 6(e) and 6(f) \\
\hline Beam energy $E_{k}(\mathrm{keV})$ & 1200 & 1100 & 1070 \\
Intersection frequency $f_{1}, f_{2}(\mathrm{GHz})$ & $73.2,32.3$ & $55.8,37.0$ & $\sim 44$ \\
Simulation $(\mathrm{FWHM}) t_{s 1}(\mathrm{nsec})$ & 0.28 & 0.20 & 0.11 \\
Analytic [Eq. $(24)] t_{s 1}(\mathrm{nsec})$ & 0.290 & 0.205 & 0.0 \\
Free space $N_{w} / f_{1}(\mathrm{nsec})$ & 0.68 & 0.90 & 1.14 \\
Simulation $(\mathrm{FWHM}) \Delta f_{1} / f_{1}$ & 0.04 & 0.09 & 0.22 \\
Analytic [Eq. $(26)] \Delta f_{1} / f_{1}$ & 0.047 & 0.087 & $\infty$ \\
Free space $1 / N_{w}$ & 0.02 & 0.02 & 0.02 \\
\hline \hline
\end{tabular}

radiation wave packet and a wide bandwidth. However, one can no longer use the first order Taylor expansion (23), and numerical computation of the inverse Fourier transform of (20)-(22) is required, in order to calculate the time domain field dependence of the radiation wave packet [4].

Here we demonstrate the effect of the waveguide dispersion on the radiation wave packet pulse duration and frequency bandwidth in the two-frequencies and zeroslippage (grazing) limits, by performing direct multifrequency FEL simulation with our 3D wideband spacefrequency particle simulation code WB3D $[25,26]$. The code was employed for an example based on the Israeli waveguide resonator FEL [27]: $E_{k}=1-1.5 \mathrm{MeV}, I_{0}=$ $2 \mathrm{~A}, t_{0}=1 \mathrm{psec}, B_{w}=2 \mathrm{kG}, \lambda_{w}=4.444 \mathrm{~cm}$. A longer wiggler $N_{w}=50$ was used to emphasize the effect. The fundamental transverse mode $\left(q=\mathrm{TE}_{01}\right)$ of a curved parallel plates waveguide was assumed. The waveguide has cutoff frequency $f_{\text {co }}=16.65 \mathrm{GHz}$ and effective mode area $A_{\mathrm{em}}=41.1 \mathrm{~mm}^{2}$. Figure 5 shows the solutions of the synchronism condition equation (18) as a function of the beam acceleration energy. Figure 6 displays the spectral power of the mode and the time dependent radiation field of the radiation wave packet emitted on axis for three examples: (a),(b) well spaced dispersion curves intersection points, (c),(d) near grazing, (e),(f) at grazing point.

The numerical simulation curves of Fig. 6 and the parameters comparison of Table I demonstrate the pulse narrowing and bandwidth widening effect of the dispersive waveguide on the waveform and its emission parameters, and its relation to the analytic approximation. The theoretical uniform waveform of the emitted wave packet (Fig. 4) turns out to be more gradual in the exact FEL code computation. In Table I, the computed FWHM width of the envelopes of the radiation field pulses and the FWHM bandwidths of the emission frequency lines are compared to the approximate expressions and the freespace emission expressions. It is observe that significant pulse compression and bandwidth expansion (26) is attained relative to the free-space emission. Note that the peak spectral power of the radiation wave packet is hardly dependent on the frequency and the nature of the dispersion equations solution [it is given by (6) with $\left.\sin c^{2}(\theta L / C)=M_{b}=M_{M}=1\right]$. However (for a fixed pulse energy), the instantaneous peak power goes up in inverse proportion to the pulse duration, and consequently the peak of the radiation field grows approximately in inverse proportion to the square root of the pulse compression.

The use of a waveguide to attain ultrashort pulses at zero-slippage condition can be of practical interest. It is a way to avoid pulse widening due to the slippage effect, and obtain short pulses, limited only by the $e$-beam bunch duration and the waveguide dispersion characteristics. In certain spectroscopic applications, intense short pulses are required, and spectral filtering, if needed, is done externally. In such applications, now considered for CSR radiation sources, a PB-FEL, operating in a waveguide at the zero-slippage conditions, can provide superior characteristics. Its wideband short pulse radiation would be more intense and more collimated and therefore also brighter than the "single wiggle" CSR radiation pulse.

\section{STIMULATED SUPERRADIANCE IN ELECTRON-BEAM RADIATORS}

While the stimulated emission process (laser gain) and the superradiant emission have received extensive theoretical and experimental research attention, the stimulatedsuperradiance process was hardly studied, and was not exploited in development of new radiation devices, except for a related experiment carried out at the Lebedev Institute at millimeter wavelengths with a $7 \mathrm{MeV}$ microtron [14]. One may identify the ST-SR process as the emission process in the second (radiating) wiggler section of the optical klystron [28]. Sparse experiments were carried out on the "Lasertron" concept [29] (an rf tube based on a klystron cavity, into which electron beam bunches, produced by a photocathode $e$ gun, are injected) that could have been considered a stimulated-superradiance radiation device. In the wide sense, also the basic process of electron bunch acceleration in a rf linac can be viewed as "stimulated-superradiant absorption" of the rf radiation power.

In this section we will review briefly the special features of the ST-SR radiation emission process in comparison to 
the other two coherent emission processes, and will present an optional scheme of "stimulated-superradiance FEL oscillator."

Taking now the limit of continuous periodic bunching, the output radiation power $P(L)$ depends, in general, on the input radiation power $P(0)$ and the prebunching current $I_{b}=I_{0}\left|M_{b}\left(\omega_{b}\right)\right|$. For FEL in the linear-gain (small signal) $[2,30]$ regime,

$$
\begin{aligned}
\Delta P(L)= & P(0) I_{0} F_{\mathrm{FEL}}(\theta)+P_{B} I_{0}^{2}\left|M_{b}\left(\omega_{b}\right)\right|^{2} F_{\mathrm{SR}}(\theta) \\
& +\left(P(0) P_{B}\right)^{1 / 2} I_{0}\left|M_{b}\left(\omega_{b}\right)\right| F_{\mathrm{ST}-\mathrm{SR}}(\theta) \sin \varphi
\end{aligned}
$$

where $\theta$ is the detuning parameter (7) and $\varphi$ is the phase of the density bunching relative to the input radiation field at $z=0$. The detuning functions $F_{\mathrm{FEL}}, F_{\mathrm{SR}}, F_{\mathrm{ST}-\mathrm{SR}}$ and the coefficients $P_{B}$ were derived in [2] for various kinds of FELs in all linear-gain (small signal) regimes. The first term is the conventional FEL linear-gain expression, proportional to $P(0)$ and to $I_{0}$ (assuming partial or no bunching $\left.M_{b}<1\right)$. The second and third terms are the superradiant and stimulated-superradiance emissions discussed in part I (Ref. [1]) of the article. The ST-SR emission is proportional to the radiation field and therefore scales like $[P(0)]^{1 / 2}$.

The relative significance of each of the terms depends on the radiation input power $P(0)$ and the prebunching current $I_{b}$. For fixed $I_{b}$, the first term is proportional to $P(0)$, the second term is independent of $P(0)$, the third term goes like the square root of $P(0)$. These dependencies are drawn schematically in Fig. 7. Evidently, at low input power levels (below point $A$ in Fig. 7) the superradiant emission power is dominant. As the input power grows, the stimulated-superradiance process, growing as $\left([P(0)]^{1 / 2}\right.$, starts to dominate, up to the power level corresponding to point $B$. Beyond this point, the regular FEL stimulated emission power [growing in proportion to $P(0)$ ] dominates.

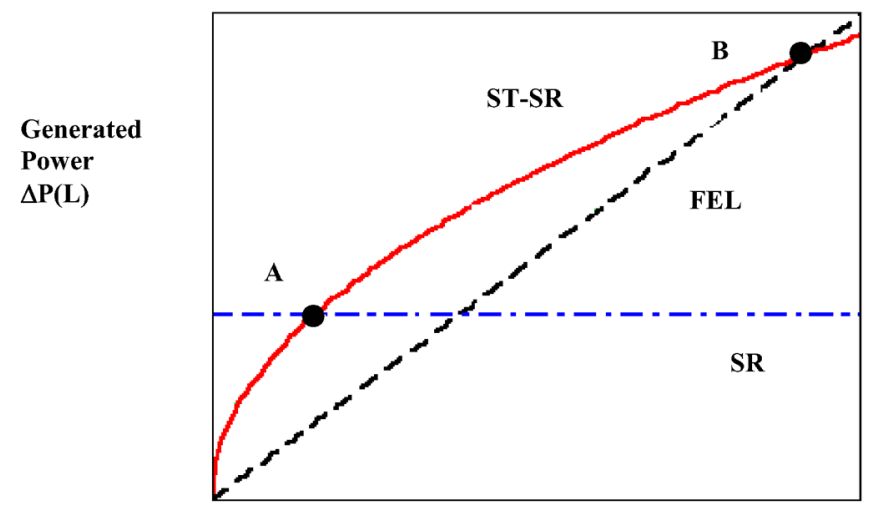

Input Power P(0)

FIG. 7. (Color) Scaling of generated power $\Delta P(L)$ versus input power $P(0)$ of the stimulated (FEL), superradiant (SR), and stimulated-superradiant (ST-SR) emission processes.
Note that some of these scaling laws, and therefore the comparative discussion, are only valid in the FEL lineargain (small signal) regime, away from FEL saturation and away from a tight bunching limit $\left[M_{b}\left(\omega_{b}\right) \cong 1\right]$. Only then the linear dependence of the first term of (30) on the input radiation power $P(0)$ and the average beam current $I_{0}$ is valid (at tight bunching condition there is no FEL gain and the first term vanishes). Furthermore, the comparison between the terms depends on the bunching frequency $\omega_{b}$. The detuning curves of the different terms are different, though they are all centered around the synchronism frequency $\omega_{0}$. In particular, in the small gain limit the FEL gain curve $F_{\mathrm{FEL}}(\theta)$ is proportional [24,31] to the derivative of $\sin c^{2}(\theta L / 2)$ (an odd function of $\omega$ - $\omega_{0}$ ) while $F_{\mathrm{SR}}(\theta)$ and $F_{\mathrm{ST}-\mathrm{SR}}(\theta)$ are proportional to the symmetric functions $\sin c^{2}(\theta L / 2)$ and $\sin c(\theta L / 2)$, respectively. Thus, for example, at $\omega=\omega_{b}=\omega_{0}(\theta=0)$ the FEL gain vanishes, and only the superradiance and stimulated-superradiance terms exist.

The scaling laws of the different terms of Eq. (30) as a function of power and bunching current were verified experimentally by Arbel et al. [30]. All three contributions to the output power were identified due to their different detuning functions. The stimulated-superradiance contribution was identified by its dependence on the phase delay $\varphi$ between the prebunching and radiation input signals.

An interesting concept of a prebunched beam FEL oscillator arises when one considers providing feedback to the ST-SR emission amplification process [32,33]. In such a device the radiation wave emitted by the bunched electron beam is intercepted inside an optical resonator of Fabri-Perot or ring configuration (see Fig. 8). The timing of the electron bunches injection into the interaction region is judicially synchronized with the round-trip time of the radiation wave inside the resonator. At steady-state saturation, a balance is attained (as in any oscillator) between the radiation generated at each path (by the process of stimulated superradiance) and the power lost inside the resonator or coupled out through the out-coupling mirror. Considering now a continuous wave situation, this balance can be stated in the form

$$
P[L ; P(0)]=P(0) / R_{\mathrm{rt}},
$$

where $P[L ; P(0)]$ is the output power at the end of the

$$
\mathrm{P}(0)=\mathrm{R}_{\mathrm{rt}} \mathrm{P}(\mathrm{L}) \quad \mathrm{P}(\mathrm{L}) \quad \mathrm{P}_{\text {out }}=\mathrm{TP}(\mathrm{L})
$$
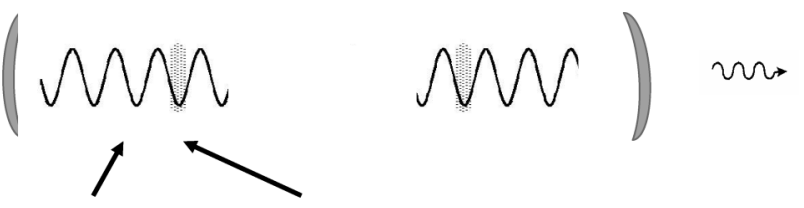

Wave packet e-bunch

FIG. 8. The ST-SR oscillator in a Fabri-Perot resonator. 
interaction length $z=L$. In the saturation regime it is a nonlinear function of $P(0)$. For a given round-trip powerreflectivity factor $R_{\mathrm{rt}}$ (see Fig. 8), the in-cavity saturation powers $P(0), P(L)$ are the solutions of (31). The outcoupled radiation power $P_{\text {out }}=T P(L)$ is then determined by the out-coupling mirror power transmission factor $T$ [by definition $R_{\mathrm{rt}} \equiv(1-\mathrm{L})(1-T)$, where $\mathrm{L}$ is the round-trip internal cavity loss factor].

The oscillation buildup and saturation process of the STSR prebunched beam FEL oscillator were analyzed numerically in Refs. [32,34]. At saturation the radiation field inside the cavity is built up, and the small signal assumptions leading to Eqs. (2) and (30) are not valid. In this article we describe the electron dynamics in the combined wiggler and radiation wave fields (the ponderomotive wave) in terms of the pendulum model [35],

$$
\begin{gathered}
\frac{d^{2} \Psi}{d z^{2}}=-K_{s}^{2} \sin \Psi, \\
\theta=\frac{d \Psi}{d z}
\end{gathered}
$$

where $\Psi(z)$ is the phase of the electron relative to the ponderomotive wave, $\Psi(z)=\int_{0}^{z} \theta\left(\omega, z^{\prime}\right) d z^{\prime}+\varphi$, and the detuning parameter $\theta(\omega, z)$ is still given by (7), except that now, because of the strong interaction with the wave, $v_{z}=$ $v_{z}(z)$ and $\theta=\theta(z)$. The electron slows down along the interaction length (corresponding to loss of energy) and the detuning parameter (7) grows respectively. The synchrotron oscillation wave number $K_{s}$ is given by

$$
\begin{gathered}
K_{s}=\frac{k \sqrt{a_{w} a_{s} / 2}}{\gamma \gamma_{z} \beta_{z}^{2}}, \\
a_{s}=\frac{e\left|\tilde{\mathbf{E}}_{\perp}\right|}{m c \omega}=\frac{e}{m c \omega}\left(\frac{2 Z_{q} P}{A_{\mathrm{em}}}\right)^{1 / 2},
\end{gathered}
$$

where $\mathbf{E}$ and $P$ are the circulating radiation field and power in the saturating oscillator cavity (we assume high roundtrip resonator reflectivity, and therefore constant power $-P$ along the resonator).

As is well known, the pendulum equation (32) can be integrated once, resulting in a picture of open and closed trajectories in $\theta-\Psi$ phase space (Fig. 9). This picture can also be viewed as a display of electron energy versus phase trajectories, if one uses the differential linear relation between $\gamma$ and $\theta$ near the synchronism energy $\gamma_{0}$,

$$
\begin{aligned}
\delta \gamma(z) & =\gamma(z)-\gamma_{0}=\theta(z) /\left(\frac{d \theta}{d v_{z}} \frac{d v_{z}}{d \gamma}\right) \\
& =-\beta_{z_{0}}^{3} \gamma_{z_{0}}^{2} \gamma_{0} \frac{\theta(z)}{k}
\end{aligned}
$$

where $\gamma_{0}$ corresponds to the energy of a synchronous electron, for which $\theta\left(\omega, v_{z 0}\right)=0$. Whether an electron executes along the interaction length an open or closed

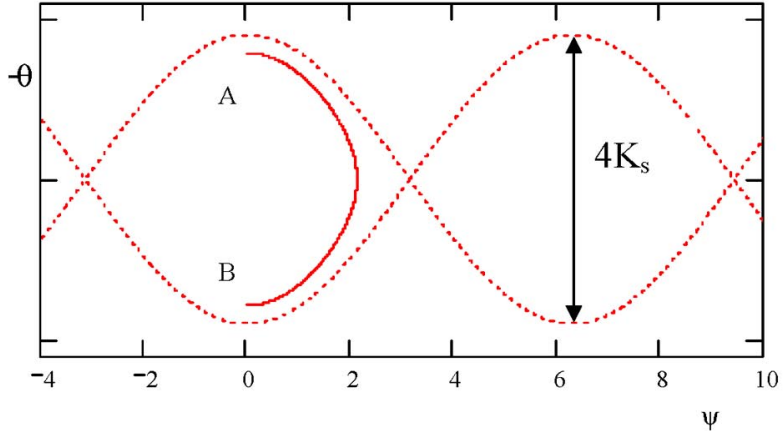

a)

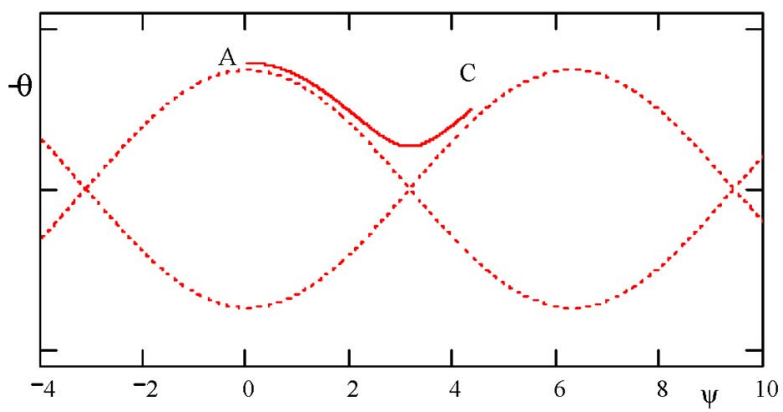

b)

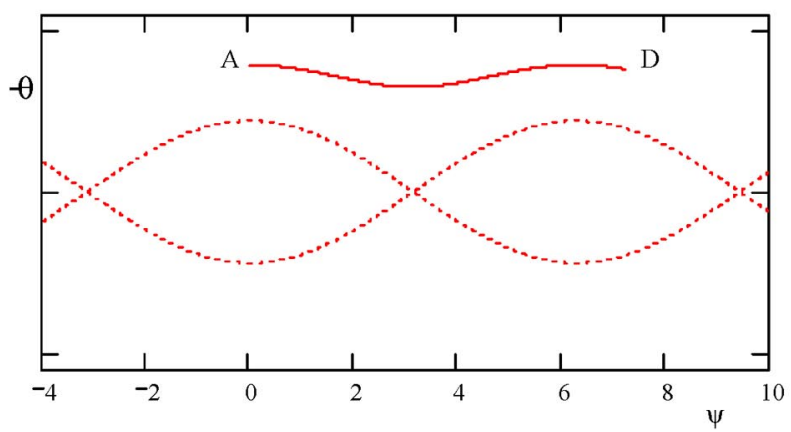

c)

FIG. 9. (Color) Closed (a) and open (b),(c) electron saturation trajectories in the prebunched beam FEL oscillator in different stored circulating power conditions (different values of $K_{s}$ ). In all cases the electron starts at point $A: \Psi(0)=0$. In (a) the electron bunch is initially inside the separatrix $\left[2 K_{s}>-\theta(0)\right]$, it stays trapped and arrives to maximal energy loss at point $B$ : $\theta(L)=-|\theta(0)|$. In (b) and (c) the electron bunch is initially outside the separatrix $\left[2 K_{s}<-\theta(0)\right]$ and stays in open trajectories, up to points $C$ and $D$, losing in both cases less energy than in case (a).

trajectory at steady state depends on the initial detuning conditions $\theta(0)$ [or $\delta \gamma(0)=\gamma(0)-\gamma_{0}$ ] and initial relative phase $\Psi(0)=\varphi$. Three examples are shown in Fig. 9: when the initial condition coordinates are inside the "separatrix" (a), the electron follows a closed (trapped) trajectory inside the ponderomotive potential "bucket" (trap). When starting out of the separatrix (b),(c) the trajectories are open. The separatrix is given by the equation

$$
\theta(\Psi)= \pm 2 K_{s} \cos (\Psi / 2)
$$




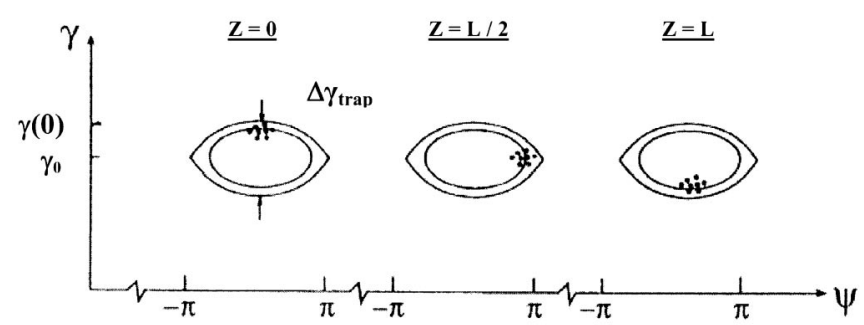

FIG. 10. Optimal (high energy extraction) saturation dynamics of an $e$-bunch in the prebunched beam FEL oscillator.

which means that the trap "depth" [Fig. 9(a)] is $(\Delta \theta)_{\text {trap }}=$ $4 K_{s}$. Examination of Fig. 9 suggests the following strategy to attain maximal radiation energy extraction from the electron bunch, as described in Fig. 10.

(i) The electron bunches should enter the interaction region at a bunching frequency $\omega_{b}$ synchronized in phase with the round-trip time of a radiation wave of frequency $\omega=\omega_{b}$. Namely, the bunching frequency should match a resonant longitudinal mode of the cavity.

(ii) The bunch's arrival time into the interaction region $t_{0}$, should match the phase of the circulating radiation wave, so that each bunch will start its phase-space trajectory at $z=0$ in the center phase of the ponderomotivewave bucket.

(iii) The electron beam bunches should be injected periodically into the interaction region with initial energy $\gamma(0)>\gamma_{0}$ [corresponding to detuning parameter $\theta(0)<$ $0]$, which is close to the top of the bucket, but still inside it, namely [see (36) and (37) and Fig. 9(a)],

$$
\begin{aligned}
& -\theta(0) \lesssim(\Delta \theta)_{\text {trap }} / 2=2 K_{s}, \\
& \delta \gamma(0) \lesssim 4 \beta_{z 0}^{3} \gamma_{z 0}^{2} \gamma_{0} K_{s} / k .
\end{aligned}
$$

(iv) The design parameters ( $L, a_{w}, T$, etc.) should be adjusted so that the electron bunch will perform about half of a synchrotron oscillation along the interaction length. This way the electrons will reach the bottom of the bucket and release to the radiation field the maximum energy they can: $\Delta \gamma=2 \delta \gamma(0)$. When $|\theta(0)| \ll 2 K_{s}$, namely, the bunch is deep inside the trap right at entrance $(z=0)$, the synchrotron oscillation period is $2 \pi / K_{s}$, and the design parameters for attaining half a synchrotron oscillation should be chosen to satisfy

$$
K_{s} L=\pi .
$$

However, when we want to extract more energy by keeping $-\theta(0)$ at the top of the trap [Eq. (38)], the synchrotron oscillation period $2 \pi / K_{s}^{\prime}$ becomes longer than $2 \pi / K_{s}$ $\left(K_{s}^{\prime}>K_{s}\right)$ and the resonator parameters should be designed to attain the maximum energy extraction $(\Delta \gamma)_{\text {trap }}=$ $2 \delta \gamma(0)$ at an optimal parameter value $K_{s}^{\prime} L=\pi$ instead of (39).

(v) The energy bunching should be tight enough [satisfying $\left.\left\langle\left[\omega\left(t_{0 j}-t_{0}\right)\right]^{2}\right\rangle^{1 / 2} \ll 2 \pi\right]$ and cold enough $[\langle[\gamma-$ $\left.\gamma(0)]\rangle^{1 / 2} \ll 4 \beta_{z 0}^{3} \gamma_{z 0}^{2} \gamma_{0} K_{s} / k\right]$, in order that all electrons in the bunch will follow the same trajectories and emit coherently the same maximal radiative energy.

When these conditions are met, all electrons in the bunch detuned as in (38) lose the same amount of energy in favor of the radiation field,

$$
2 \delta \gamma(0) \lesssim(\Delta \gamma)_{\text {trap }} \cong 8 \beta_{z 0}^{3} \gamma_{z 0}^{2} \gamma_{0} K_{s} / k,
$$

and the maximum radiation power that can be generated by the oscillator at steady-state condition is

$$
(\Delta P)_{\max } \cong \frac{I_{0}}{e}(\Delta \gamma)_{\text {trap }} m c^{2} \cong 8 I_{0} \frac{m c^{2}}{e} \beta_{z} \gamma_{z} \sqrt{a_{w} a_{s} / 2}
$$

Equations (40) and (41) are upper limit estimates of the energy and power extraction levels possible with a tightly bunched beam. However, this limit can never be attained, because if we try to set $-\theta(0)$ to be as close as possible to $2 K_{s}$, the modified synchrotron oscillation period $2 \pi / K_{s}^{\prime}$ becomes infinitely long and the electron does not arrive to the bottom of the trap at the end of the wiggler. To find out correctly the energy extraction efficiency and power, one should compute $\theta(z)$ numerically and calculate the extraction efficiency [based on (36)],

$$
\begin{aligned}
\eta_{\mathrm{ext}} & =\frac{\left\langle\gamma_{i}(0)-\gamma_{i}(L)\right\rangle}{\gamma-1} \\
& =\frac{\gamma}{\gamma-1} \frac{\beta_{z}^{3} \gamma_{z}^{2}}{k}\left\langle-\left[\theta_{i}(0)-\theta_{i}(L)\right]\right\rangle,
\end{aligned}
$$

where the averaging $\langle\cdots\rangle$ is over all the electrons in the bunch (in a tightly bunched electron beam the averaging symbol can be omitted). This can be expressed in terms of normalized universal parameters,

$$
\begin{gathered}
\eta_{\mathrm{ext}}=\frac{\gamma}{\gamma-1} \beta_{z}^{3} \gamma_{z}^{2} \frac{\lambda}{2 L} \eta_{\theta} \cong \frac{1}{4 N_{w}} \eta_{\theta}, \\
\eta_{\theta}=\frac{-\left\langle\bar{\theta}_{i}(0)-\bar{\theta}_{i}(1)\right\rangle}{\pi},
\end{gathered}
$$

and $\bar{\theta}_{i}(\bar{z})$ is the solution of the normalized pendulum equation for $0 \leq \bar{z} \leq 1$,

$$
\begin{gathered}
\frac{d \bar{\theta}_{i}}{d \bar{z}}=\bar{K}_{s}^{2} \sin \Psi_{i}, \\
\frac{d \Psi_{i}}{d \bar{z}_{i}}=\bar{\theta}_{i} .
\end{gathered}
$$

These equations are equal to (32) and (33) with the substitutions $\bar{z}=z / L, \bar{\theta}_{i}(\bar{z})=\theta_{i}(z) L, \bar{K}_{s}=K_{s} L$. The second part of (43) is valid only in the highly relativistic limit $\left(\gamma_{z} \gg 1\right)$ and free-space wave propagation.

Figure 11(a) displays the normalized extraction efficiency $\eta_{\theta}$ as a function of $-\bar{\theta}_{0}$ for various values of $\bar{K}_{s}$ for a tightly bunched electron beam. The horizontal broken 
(a)

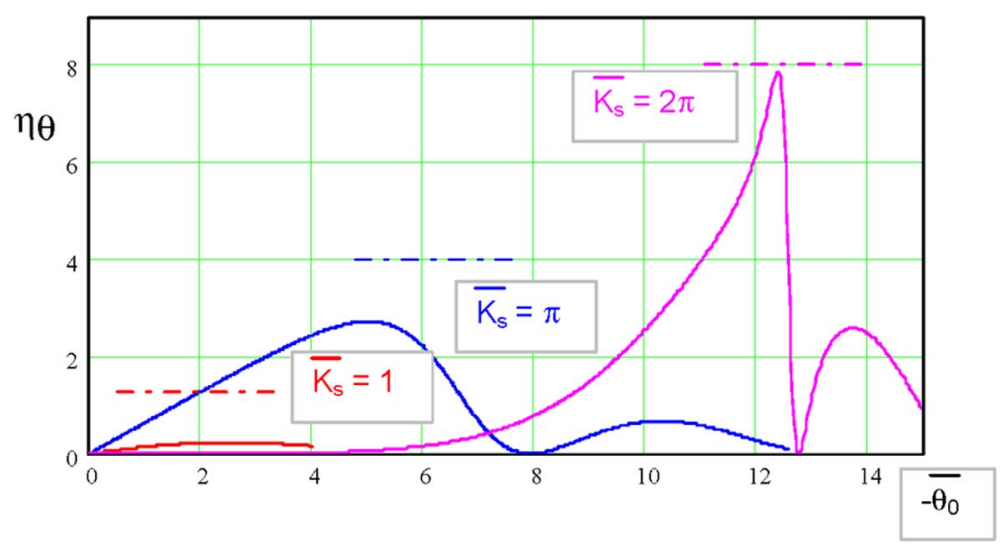

(b)

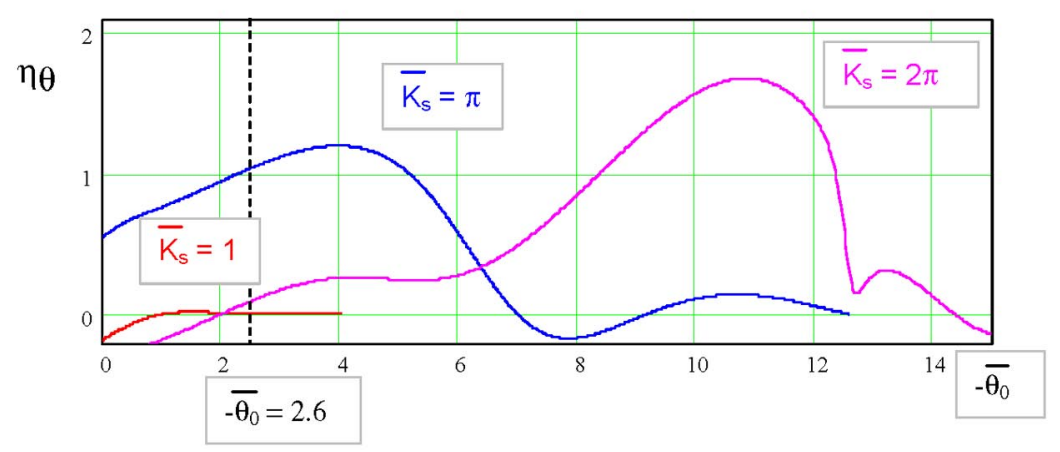

FIG. 11. (Color) Normalized extraction efficiency $\eta_{\theta}$ [Eq. (44)] as a function of initial detuning $\bar{\theta}(0)$ for a prebunched FEL (a) and a conventional FEL (b).

lines indicate the upper limit value of $\eta_{\theta}$, corresponding in (44) to $\bar{\theta}(1)=-\bar{\theta}(0)=2 \bar{K}_{s}(38)$ :

$$
\eta_{\theta, \max }=4 \bar{K}_{s} / \pi
$$

We see from Fig. 11(a) that this upper limit can be approached [and then the estimates (40) and (41) are valid] only when $\bar{K}_{s} \gg \pi$.

For comparison we show in Fig. 11(b) the value of the normalized efficiency $\eta_{\theta}$ for a conventional FEL with uniform initial electron phase distribution. Note that for $\bar{K}_{s}=\pi$ and initial detuning $\bar{\theta}(0)=-2.6$ (corresponding to FEL maximum small signal gain), $\eta_{\theta} \cong 1$ and consequently the commonly accepted FEL extraction efficiency expression $\eta_{\text {ext }}=1 / 4 N_{w}$ is recovered in (43). Also note that when $\bar{K}_{s} \gg 1$ the prebunched beam FEL is more efficient. For $\bar{K}_{s}=2 \pi$ its maximal efficiency is fivefold bigger than the conventional FEL efficiency. In general, the extraction efficiency enhancement factor relative to the FEL expression $1 / 4 N_{w}$ is given in this limit by (47).

From this discussion it turns out that the power generation $P_{\text {gen }}$ in the PB-FEL oscillator may approach the maximum value (41) when $\bar{K}_{s} \gg \pi$, and then the maximum attainable radiative extraction efficiency is

$$
\eta_{\text {ext }}=\frac{P_{\text {gen }}}{P_{\text {beam }}}=\frac{\gamma \gamma_{z}^{3} \beta_{z}^{2}}{\gamma-1} \frac{\lambda}{L} \frac{2 \bar{K}_{s}}{\pi} \cong \frac{\bar{K}_{s}}{\pi} \frac{1}{N_{w}},
$$

where the last equality is valid for the highly relativistic limit $(\gamma \gg 1)$. This value is a factor $\times 4 \bar{K}_{s} / \pi \gg 1$ larger than the extraction efficiency of a conventional FEL [18]. The reason is clearly related to the possibility to form a deep trap, place all electrons of a tight bunch at the top of the trap, and extract the maximum energy $\Delta \gamma_{\text {trap }}$ from all of them (Fig. 10).

The question still remains of whether the dynamics of the oscillation buildup in a PB-FEL oscillator can bring about steady-state operation according to the ideal scheme of Fig. 10 and attain the maximal extraction efficiency (48). This problem was analyzed numerically in [32], where it was first identified that this special kind of oscillator has bistable characteristics. Possibilities of bistable operation were identified also in other FEL based systems [36]. In the case of a PB-FEL oscillator it may cause some difficulty to attain the maximum efficiency and power. We extend here the analysis in [32] of PB-FEL oscillator, and correct some earlier errors.

It is convenient to present the oscillation buildup process in terms of normalized power $p=\bar{K}_{s}^{4}$ and normalized incremental gain $g=\eta_{\theta} / \bar{K}_{s}^{4}$ parameters,

$$
\begin{gathered}
P=A_{p} p=A_{p} \bar{K}_{s}^{4}, \\
G-1 \equiv \frac{\Delta P}{P}=A_{g} \frac{\eta_{\theta}}{\bar{K}_{s}^{4}},
\end{gathered}
$$

where from (34) and (35), 


$$
A_{p}=\frac{1}{2 \pi^{2}} \cdot \frac{\left(\gamma \gamma_{z} \beta_{z}^{2}\right)^{4}}{a_{w}^{2}} \cdot \frac{A_{\mathrm{em}} \lambda^{2}}{L^{4}} \cdot \frac{\left(m c^{2} / e\right)^{2}}{Z_{q}},
$$

and from conservation of power $\Delta P=P_{\text {gen }}=\frac{I_{0}}{e} \times$ $(\gamma-1) m c^{2} \eta_{\text {ext }}$ and Eq. (43),

$$
A_{g}=\pi^{2} \frac{a_{w}^{2}}{\gamma^{3} \gamma_{z}^{2} \beta_{z}^{5}} \cdot \frac{L^{3}}{A_{\mathrm{em}} \lambda} \cdot \frac{Z_{q} I_{0}}{m c^{2} / e}
$$

The oscillation buildup process in a resonator of round-trip reflectivity $R_{\mathrm{rt}}$ is expressed by

$$
P_{n+1}=\left(P_{n}+\Delta P_{n}\right) R_{\mathrm{rt}}
$$

(where $n=0,1,2, \ldots$ is the oscillation round-trip time of the radiation mode in the resonator), which can be written in the normalized form

$$
\bar{K}_{s n+1}^{4}=\left(\bar{K}_{s n}^{4}+A_{g} \eta_{\theta n}\right) R_{\mathrm{rt}} .
$$

The parameter $\eta_{\theta n}$ is calculated in each step from (44) based on the solution of the normalized pendulum equation (45) and (46) for fixed initial detuning value $\bar{\theta}_{0}$ and constant $\bar{K}_{s n}$ along the wiggler (low gain assumption).

At steady-state saturation $\bar{K}_{s n+1}=K_{s n}$, and (54) turns out to be a normalized version of Eq. (31). The oscillator steady-state saturation points are then the solutions of

$$
\frac{\eta_{\theta}\left(\bar{\theta}_{0}, \bar{K}_{s}\right)}{\bar{K}_{s}^{4}}=\frac{\left(1-R_{\mathrm{rt}}\right) / R_{\mathrm{rt}}}{A_{g}} .
$$

This is equivalent to equating the single path incremental gain along the interaction length to the round-trip losses in the resonator, which is the conventional oscillation condition of lasers. Since $\eta_{\theta}$ can be calculated in general in terms of the normalized parameters $\bar{\theta}_{0}, \bar{K}_{s}$ by solving the pendulum equation (45) and (46) [see Eq. (44) and Fig. 11], the significance of (55) is that the steady-state saturation condition of any PB-FEL can be determined (for a given initial detuning parameter value $\bar{\theta}_{0}$ ) by a single "feedback parameter" $\left(R_{\mathrm{rt}}^{-1}-1\right) / A_{g}$.

Figure 12 displays the graphical solution of (55) as the intersection between the normalized incremental gain curve $\eta_{\theta} / \bar{K}_{s}^{4}$ calculated for $-\bar{\theta}_{0}=9.425>2 \pi$ and the constant parameter $\left(R_{\mathrm{rt}}^{-1}-1\right) / A_{g}$. The figure displays the bistability nature of the PB-FEL for feedback-parameter value 0.005 . Analysis of the curve reveals that only the first and third solutions (intersections) are stable. The second one is unstable. When the feedback-parameter value is $\left(R_{\mathrm{rt}}^{-1}-1\right) / A_{g}=0.01$, there is only a single steady-state solution. Note that in any case the PB-FEL oscillator has no oscillation threshold. Because the stimulatedsuperradiance differential gain $\Delta P / P \propto P^{-1 / 2}$ explodes at low power (small values of $\bar{K}_{s}$ ), there is always a solution (though at low power values) to the oscillation condition (55).

The (red line) contours $B, C, D$ in Fig. 13 are another display of the geometrical contour [in plane $\left.\left(\bar{K}_{s}, \bar{\theta}_{0}\right)\right]$ of the

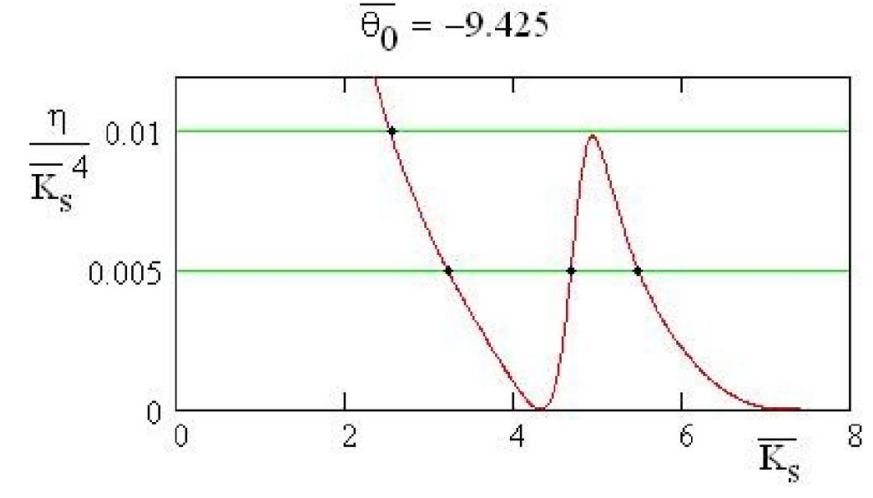

FIG. 12. (Color) Solutions of the steady-state oscillation condition (55). For $\left(R_{\mathrm{rt}}^{-1}-1\right) / A_{g}=0.01$ there is single stable solution. For $\left(R_{\mathrm{rt}}^{-1}-1\right) / A_{g}=0.005$ the intersections correspond to the two bistable solutions and a third unstable solution in between.

oscillator stable operation points [solutions of Eq. (55)], drawn for a particular value of the feedback parameter $\left(R_{\mathrm{rt}}^{-1}-1\right) / A_{g}=0.005$. The branch $B$ corresponds to saturation at the desirable closed trajectories (trapping) points [see Fig. 9(a)]. It is entirely above the diagonal line (representing the separatrix) $-\bar{\theta}_{0}<2 \bar{K}_{s}$. Namely, at steady state $B$, fresh bunches enter into the trap right upon entrance to the interaction region $(\bar{z}=0)$, and perform somewhat more than half an oscillation period, in order to release radiation power that will keep the steady state balance between generation and loss in the oscillator. The branch $D$ corresponds to the "open-trajectories saturation" process described in Fig. 9(c). This stable steadystate branch (and saturation process) exist only in the parameters regime $2 \pi<-\bar{\theta}_{0}<4 \pi$ (for higher values of $-\bar{\theta}_{0}$ there are more branches of stable open trajectories corresponding to slippage of the bunch over more than one ponderomotive wave trap). The branch $C$ is a branch of unstable equilibrium oscillator working points. Namely,

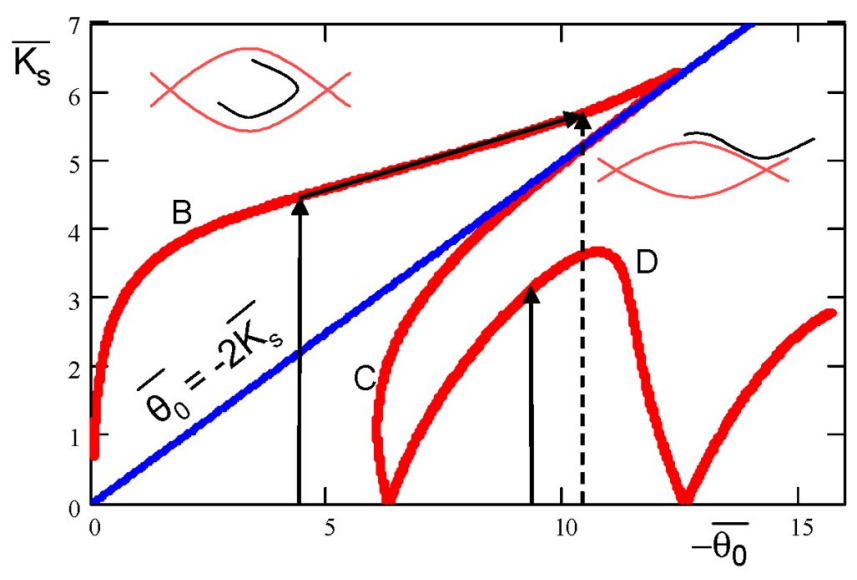

FIG. 13. (Color) Representation of the steady-state saturation contours of the stimulated-superradiance FEL oscillator in $\left(\bar{K}_{s}, \bar{\theta}_{0}\right)$ parameters plane for $\left(R_{\mathrm{rt}}^{-1}-1\right) / A_{g}=0.005$. 
any disturbance from these points (for fixed $\bar{\theta}_{0}$ ) drifts the oscillator to stable equilibrium in branch $B$ or $D$.

In order to examine the oscillation buildup process up to saturation and the bistable nature of the oscillator, we calculate the sequential oscillation buildup equations (54) for many resonator round-trips. Starting from given initial normalized power and detuning parameters $\left(\bar{\theta}_{0}, \bar{K}_{s 0}\right)$, calculate the updated $\bar{K}_{s n+1}$ of round-trip $n+1$ by solving numerically the normalized pendulum equations (45) and (46) and employing Eqs. (44) and (54). Video 1 displays the oscillation buildup process for $-\bar{\theta}_{0}=5.5<2 \pi$, starting from an arbitrary small value of $\bar{K}_{s 0}=0.001$ up to the stable "trapped electrons" saturation point on branch $B$. Video 1(b) displays the growth of the separatrix and the bunch phase-space trajectories evolution in consecutive

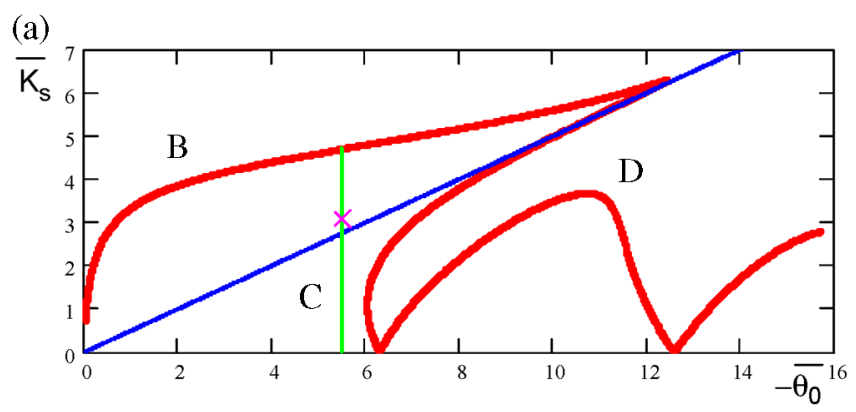

(b)

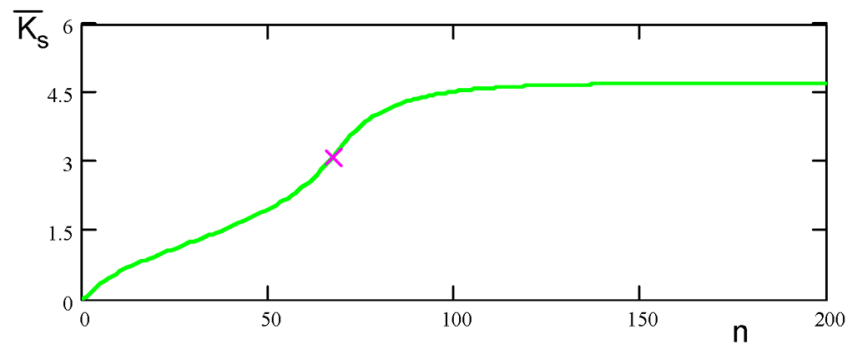

(c)

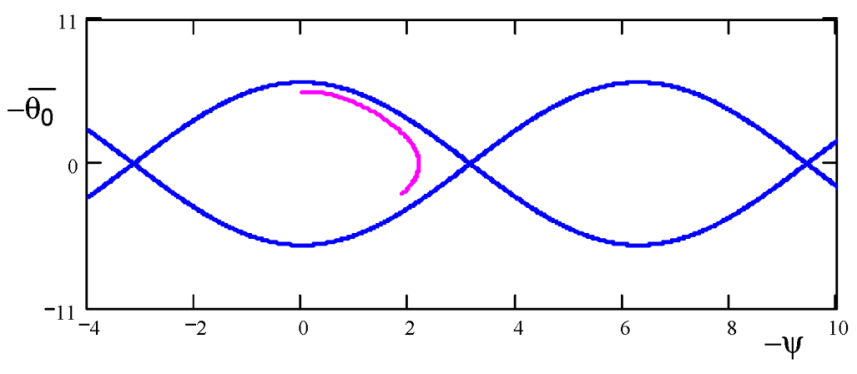

VIDEO 1. Dynamics of oscillation buildup in a stimulatedsuperradiant FEL oscillator in a single stable point operating regime $\left(\bar{\theta}_{0}=-5.5<-2 \pi\right)$. (a) The growth of $\bar{K}_{s} \propto P^{1 / 4}(P$ is the circulating power) with time up to stable saturation. (b) $\bar{K}_{s}$ as a function of the resonator circulation round-trip number. (c) Evolution of the separatrix (the trap) and the tight electron bunch trajectory as a function of time ending with trapping near the bottom of the trap. round-trips. Video 1(c) displays the growth of $\bar{K}_{s}$ during this process (in these simulations $A_{g}=6.186, R_{\mathrm{rt}}=0.97$ ).

We note that in practice there is no problem in starting this oscillation buildup process from null radiation field. Contrary to all laser oscillator, the oscillation does not start from noise (which theoretically is absent in PB-FEL) but from superradiant radiation. This, and the high gain associated with the stimulated-superradiance process at low power levels, is responsible for the quick (initial oscillation buildup process of this kind of oscillator demonstrated by simulation in Video 1(c).

Videos 2-4 illustrate the dynamics of the oscillation buildup in the bistable operating region $2 \pi<-\bar{\theta}_{0}<4 \pi$.

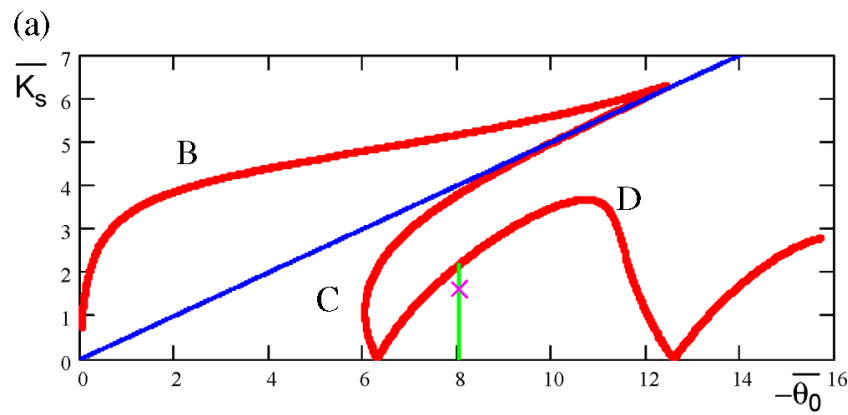

(b)

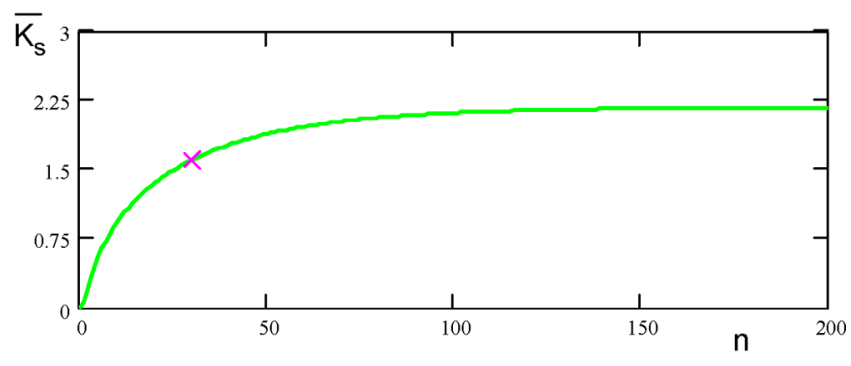

(c)

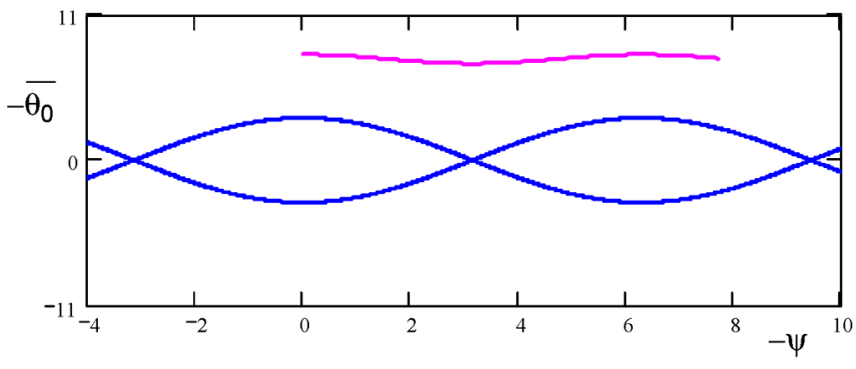

VIDEO 2. Dynamics of the oscillation buildup in the bistable operating regime: Starting from low power for $\bar{\theta}_{0}=-8$, the oscillator saturates at the first (low) stable saturation point. The bunch trajectories are open all along, and end up at saturation out of the traps beyond the second maximum. (a) The growth of $\bar{K}_{s} \propto P^{1 / 4}$ ( $P$ is the circulating power) with time up to stable saturation. (b) $\bar{K}_{s}$ as a function of the resonator circulation round-trip number. (c) Evolution of the separatrix (the "trap") and the tight electron bunch trajectory as a function of time ending up without trapping. 
(a)

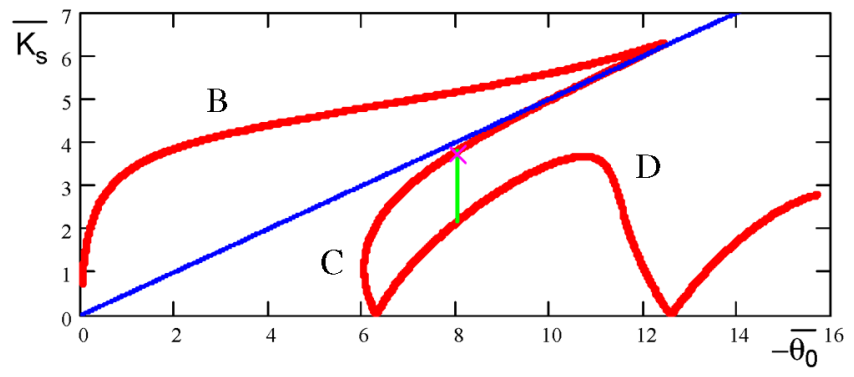

(b)

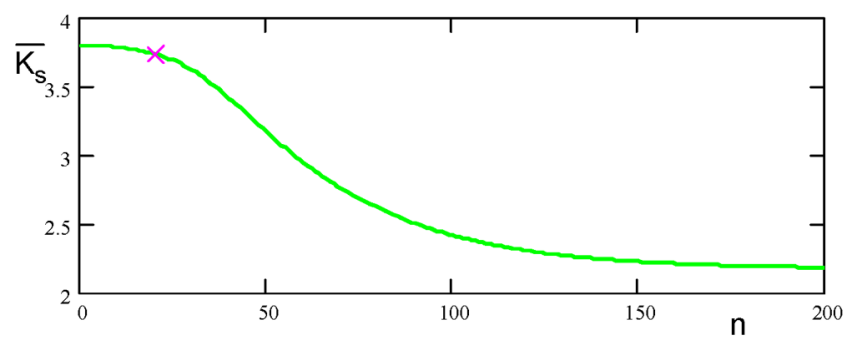

(c)

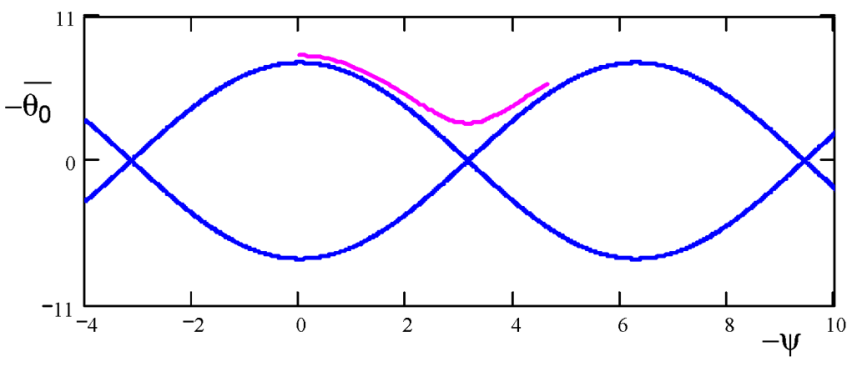

VIDEO 3. Dynamics of oscillator stored power decay in the bistable operating regime. Starting from a power level slightly below the unstable equilibrium point, the oscillator power decays and it ends up at the low stable saturation point. (a) The growth of $\bar{K}_{s} \propto P^{1 / 4}$ ( $P$ is the circulating power) with time up to stable saturation. (b) $\bar{K}_{s}$ as a function of the resonator circulation round-trip number. (c) Evolution of the separatrix (the trap) and the tight electron bunch trajectory as a function of time ending up without trapping.

In these three examples $\bar{\theta}_{0}=-8$. When one starts from low resonator power $\bar{K}_{s 0}=0.001$ (Video 2) the oscillator arrives to a stable steady-state point on the "open trajectories branch" $D$. Also when one starts from a high resonator power level $\left(\bar{K}_{s 0}=3.8\right)$, which is, however, below the unstable steady-state branch $C$, the oscillator settles back to a low power level working point on branch $D$ (Video 3). Only when the initial resonator power exceeds the unstable steady-state branch $C\left(\bar{K}_{s 0}=3.9\right)$ (Video 4), the oscillator power grows up and saturates at a high power stable steady-state point on branch $B$.

Evidently, in order to attain the high efficiency and high power advantage of the PB-FEL (41) and (48) it is best to operate in the regime $-\bar{\theta}_{0} \cong\left(2 K_{s}\right)_{\text {sat }} \gg 2 \pi$. However, to

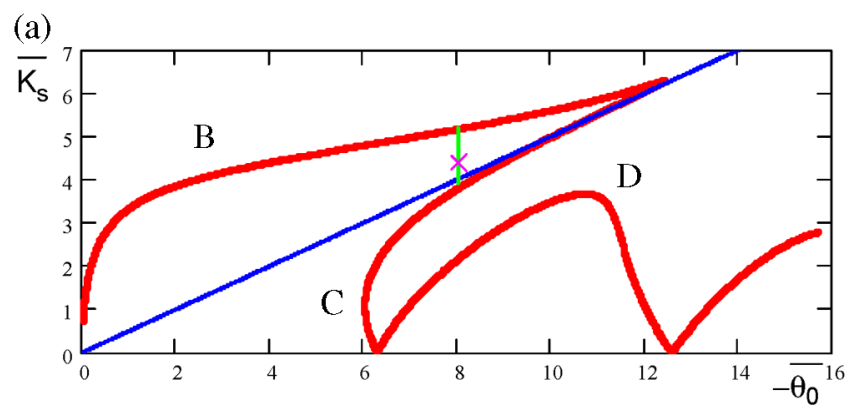

(b)

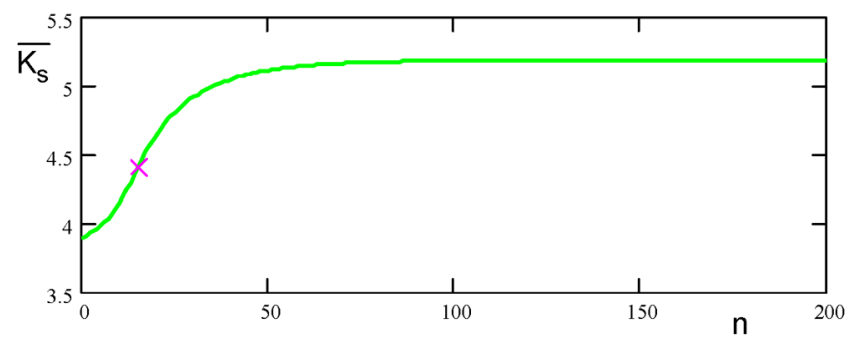

(c)

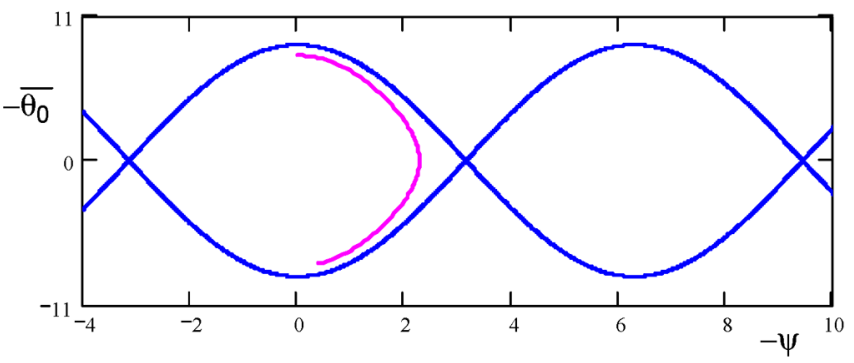

VIDEO 4. Dynamics of oscillator buildup in the bistable operating regime. Starting from a power level slightly above the unstable equilibrium point, the oscillator power grows and it ends up at the high stable saturation point. (a) The growth of $\bar{K}_{s} \propto P^{1 / 4}$ ( $P$ is the circulating power) with time up to stable saturation. (b) $\bar{K}_{s}$ as a function of the resonator circulation round-trip number. (c) Evolution of the separatrix (the trap) and the tight electron bunch trajectory as a function of time ending with trapping near the bottom of the trap.

arrive at the desirable high-efficiency trapped-electrons stable branch $B$, one must overcome the bistability problem and "leap" over the "open-trajectories" stable saturation branch $D$. One obvious way to do it is to inject into the resonator initial power with a corresponding $K_{s 0}$ parameter, which exceeds the unstable steady-state branch $D$. Another, probably more practical scheme, would be to start with low value of detuning parameters $-\bar{\theta}_{0}$, which will bring the oscillator to a stable saturation point on branch $B$ (as in Video 1), and then employ a postsaturation process of temporal tapering of the detuning parameter (by gradual increase of the $e$-beam energy) until the maximum power or efficiency point is attained. This postsaturation detuning ramping process (Fig. 13) can be done through any adia- 
batic contouring of the beam energy, avoiding crossing of the unstable branch contour at any point. Additional numerical study will be needed in order to find the best or fastest energy contouring curve.

The efficiency advantage of the prebunched beam FEL oscillator is much more significant when an energyretrieval scheme is used (e.g., in a superconducting rf accelerator FEL [37] or in an electrostatic accelerator FEL $[38,39])$. In energy-retrieval schemes, part of the kinetic power of the spent electron beam is returned to the acceleration system, and thus the system needs to supply a smaller level of net power $P_{\text {acc }}<P_{\text {beam }}$ for the acceleration process. This power $P_{\text {acc }}$ is usually equal to the sum of the extracted radiation power $P_{\text {gen }}$ and power dissipated on the accelerator walls and the beam collector or beam dump. The figure of merit for radiation generation efficiency of the accelerator is then

$$
\eta_{\mathrm{gen}}=\frac{P_{\mathrm{gen}}}{P_{\mathrm{acc}}}=\eta_{\mathrm{ext}} \cdot \eta_{\mathrm{ret}}
$$

where $\eta_{\text {ret }}=P_{\text {beam }} / P_{\text {acc }}>1$ is the energy-retrieval factor.

As mentioned above, in ST-SR oscillator all the electrons in the tight electron bunch perform the same phasespace trajectories (see Fig. 10), and therefore their energy spread at the end of the interaction region is small. This is in contrast to the conventional FEL oscillator, in which the electrons enter the interaction region at random phase $-\pi<\varphi<\pi$, and therefore each follows a different trajectory, including open trajectories, and ends up with different finite energy. Consequently, the wasted beam energy spread $(\Delta \gamma)_{\text {spread }}$ in the conventional FEL is about equal to $(\Delta \gamma)_{\text {trap }}$. Collecting all the energy-dispersed electrons at the collector requires an extra power investment (which is turned into heat) that in a simple scheme can be estimated as $P_{\text {spread }} \gtrsim I_{0} \Delta \gamma_{\text {spread }} m c^{2} / e$. Thus, in conventional energy-retrieved FEL schemes, where $(\Delta \gamma)_{\text {spread }} \cong$ $\langle\gamma(L)\rangle-\gamma(0) \cong(\Delta \gamma)_{\text {trap }}$, the accelerator "radiation generation efficiency" is limited to

$$
\eta_{\text {gen }}=\frac{P_{\text {gen }}}{P_{\text {gen }}+P_{\text {spread }}}<0.5 \text {, }
$$

which could be somewhat improved only if more elaborate schemes, such as multistage collector technology, would be used.

The significant feature of the small energy spread, produced in the beam in the prebunched free-electron maser ST-SR oscillator scheme, can be taken advantage of, in both the superconducting rf accelerator and the electrostatic accelerator energy-retrieval schemes mentioned, in order to attain much higher "radiative generation" efficiency. In the superconducting rf linac the spent beam can be decelerated in the rf cavities to lower energy before being dumped, and in the electrostatic accelerator the beam can be slowed down to near zero energy, before hitting the collector, even with a single-stage collector. Consequently, in ST-SR oscillator with energy retrieval, it is possible to attain $P_{\text {spread }} \ll P_{\text {gen }}$. The radiation generation efficiency of such a device can then be close to $100 \%$,

$$
\eta_{\text {gen }} \cong 1 \text {, }
$$

which means that almost the entire net power, invested to accelerate the $e$-beam current, turns into radiation. This interesting feature of efficient energy conversion is clearly a consequence of the low entropy or small occupation of 6D phase-space volume, associated with an ideally collimated bunched beam satisfying the inequalities $\varepsilon \ll \lambda$, $t_{b} \ll 2 \pi / \omega$.

The attainment of ultimately high net radiation generation efficiency in the ST-SR FEL oscillator (58) depends only on the availability of tight electron bunching, which enables significant energy retrieval $\eta_{\text {ret }} \cong 1 / \eta_{\text {ext }}$. This is independent of whether the high enhancement in the extraction efficiency (48) for $\bar{K}_{s} \gg \pi$ is attained, and is perhaps of more important significance.

Experimental realization of a prebunched beam FEL oscillator device requires overcoming some technological difficulties. Two main problems should be addressed in attempting to realize such a scheme at high frequencies: (i) good synchronization between the bunch repetition timing and the cavity round-trip (low jitter) and (ii) attaining continuous or long pulse train of tight electron microbunches at high repetition rate frequency.

The first problem requires continued technological development in the active field of subpicosecond pulse timing and stabilization techniques. In connection to the second problem, it is noted that in order for the scheme to work, it is not essential that the electron bunching rate $\omega_{b}$ will be equal to the radiation frequency $\omega$. As we found earlier, it is also possible to operate at a subharmonic bunching frequency $\omega_{b}=\omega / n$, which relieves the technical problem of prebunching at high repetition rate frequency. There is, however, a desirable condition to inject the electron bunches at least at a rate corresponding to the round-trip circulation time of the radiation pulses in the cavity, $c / 2 L_{c}$.

One should note that subharmonic operation of the STSR oscillator is hampered by the "slippage effect" because the radiation wave packet slips through the bunch along the interaction length (as shown in Fig. 8), and the energy extracted from the bunch is distributed over the entire wave packet of duration $t_{s}$ (24) instead of one bunching period $1 / f$. Thus the incremental power in Eq. (50) would be reduced by a factor $f \times t_{s}$, and consequently the normalization gain factor (52) is modified into

$$
A_{g}=\pi^{2} \frac{a_{w}^{2}}{\gamma^{3} \gamma_{z}^{2} \beta_{z}^{5}} \cdot \frac{L^{3}}{A_{\mathrm{em}} \lambda} \cdot \frac{Z_{q} Q_{b} / t_{s}}{m c^{2} / e},
$$

where $Q_{b}=e N_{b}$ is the bunch charge, and $P$ in (49) refers then to the wave packet peak power. In an open resonator (27) the slippage time is $t_{s}=N_{w} / f$, and thus the reduction in the single pass power increment and gain of the circu- 
lating wave packet is $N_{w}$, which may be quite significant. A possible way to increase $\bar{K}_{s}$ and arrive to higher extraction efficiency in this case is to base the oscillator on a dispersive waveguide resonator, and operate it at the zeroslippage condition (29) discussed in Sec. II B. Another possible remedy is to produce macropulses of multiple microbunches at the radiation ( $\sim \mathrm{THz})$ frequency [40], keeping the macropulses repetition rate matched to the cavity round-trip time.

Further study of the prebunched FEL oscillator is of basic interest because this device can exhibit applications of the new concept of stimulated-superradiant emission and attain ultimate efficiency of radiative energy conversion and a theoretically diminished shot noise level. In addition, the prebunched beam oscillator may raise practical interest in certain specialized situations. The most important feature of this device, which gives it an advantage over a conventional FEL oscillator, is the possibility to attain total radiation generation efficiency, close to the ultimate $\eta_{\text {gen }} \cong 1$. Examples of specialized situations where such a feature is of great importance are (i) space based radiation sources [41], where heat generation due to inefficient radiative generation generates a difficult heat dissipation problem, and (ii) future high power (MW level) radiation sources, where low radiation generation efficiencies would cause difficult heat dissipation problems and other beam energy dumping technical problems [42].

We note that further numerical studies are desirable in order to find the optimal operating conditions of this device. Use of full FEL simulation codes is preferable because the pendulum model may exhibit some effects of deviations from the exact simulation [43]. Yet previous checks of the simulation results of the PB-FEL oscillator [32] with an exact FEL numerical code FEL3D [44] did not produce significantly different results in the specific examples checked. In future work we will adopt a more accurate model for the process and avoid the assumption that the circulating power is constant along each one-way traversal. Such an improved model is necessary, especially when single bunches are considered, and the effect of the wave packet slippage modifies substantially the circulating radiation waveform.

Finally, it is pointed out that an ST-SR oscillator employing a tapered wiggler [45] can exhibit very high power emission in addition to high efficiency. In this scheme, the phase velocity of the ponderomotive wave, which traps the electron bunches, slows down along the wiggler relative to the axial velocity of the $e$-beam due to up-shift tapering of the wiggler wave number $k_{w}(z)$, or down-shift tapering of the wiggler magnetic field intensity $B_{w}(z)$. The electron bunches, trapped in the slowing down buckets, would then be forced to emit more radiation corresponding to the energy shift of the trap without $e$-beam energy spread. Without tight bunching, such a scheme cannot operate at high average power level, because the large energy spread, caused by untrapped electrons, would make it impossible to employ effective energy retrieval. Such a tapered wiggler prebunched-beam FEL oscillator combined with an energy-retrieval scheme is conceivable, but has not yet been studied experimentally.

\section{CONCLUSIONS}

In the first part of a two-parts article, a general formulation for superradiant and stimulated-superradiant radiation emission processes in prebunched electron-beam radiation schemes was derived. Special attention was given to the comparison between prebunched FEL and coherent synchrotron radiation.

In the present article, we presented schemes for enhancing SR and ST-SR emission from prebunched beam radiators. In particular, the enhancement of SR emission in a waveguide PB-FEL was analyzed, and a new scheme of ST-SR oscillator, based on a prebunched beam FEL, was analyzed. This kind of radiation source has been demonstrated experimentally, but hardly studied and optimized. It is expected to possess an important feature of ultimately high radiative energy conversion efficiency.

\section{ACKNOWLEDGMENTS}

This work was performed in the Israeli FEL National Knowledge Center supported by the Ministry of Science, Israel, and was supported in part by the Ministry of Infrastructure.

[1] A. Gover, Phys. Rev. ST Accel. Beams 8, 030701 (2005).

[2] I. Schnitzer and A. Gover, Nucl. Instrum. Methods Phys. Res., Sect. A 237, 124 (1985).

[3] F. Ciocci, R. Bartolini, A. Doria, G. P.Gallerano, M.F. Kimmitt, G. Messina, and A. Renieri, Phys. Rev. Lett. 70, 928 (1993).

[4] A. Gover, F. V. Hartemann, G. P. Lesage, N. C. Luhmann, R. S. Zhang, and C. Pelllegrini, Phys. Rev. Lett. 72, 1192 (1994).

[5] B. Faatz, A. A. Fateev, J. Feldhaus, J. Krzywinski, J. Pflueger, J. Rossbach, E. L. Saldin, E. A. Schneidmiller, and M. V. Yurkov, Nucl. Instrum. Methods Phys. Res., Sect. A 475, 363 (2001).

[6] A. Doria, R. Bartolini, J. Feinstein, G.P. Gallerano, and R. H. Pantell, IEEE J. Quantum Electron. 29, 1428 (1993).

[7] M. Cohen, A. L. Eichenbaum, M. Arbel, D. Ben-Haim, H. Kleinman, M. Draznin, A. Kugel, I. M. Yakover, and A. Gover, Phys. Rev. Lett. 74, 3812 (1995).

[8] S. Mayhew, A. I. Al-Shamma'a, R. A. Stuart, A. Shaw, C. Balfour, and J. Lucas, Nucl. Instrum. Methods Phys. Res., Sect. A 393, 356 (1997).

[9] Y. U. Jeong, Y. Kawamura, K. Toyoda, C. H. Nam, and S. S. Lee, Phys. Rev. Lett. 68, 1140 (1992).

[10] M. Asakawa, N. Sakamoto, N. Inoue, T. Yamamoto, K. Mima, S. Nakai, J. Chen, M.Fujita, K. Imasaki, C. Yamanaka, N. Ohigash, T. Agari, T. Asakuma, and Y. 
Tsunawaki, Nucl. Instrum. Methods Phys. Res., Sect. A 341, 72 (1994).

[11] http://www.frascati.enea.it/thz-bridge/fel-cats.htm

[12] R. Bonifacio, C. Maroli, and N. Pivola, Phys. Rev. A 40, 4467 (1989).

[13] A. Gover, A. Friedman, and A. Luccio, Nucl. Instrum. Methods Phys. Res., Sect. A 259, 163 (1987).

[14] N. S. Ginzburg and A. S. Sergeev, Opt. Commun. 91, 140 (1992).

[15] G. L. Carr, M. C. Martin, W. R. McKinney, K. Jordan, and G. R. Neil, Nature (London) 420, 153 (2002).

[16] M.A. Piestrup and P.F. Finman, IEEE J. Quantum Electron. 19, 357 (1983).

[17] A. Yariv, Quantum Electronics (Wiley, New York, 1988).

[18] A. Gover , H. Freund, V. L. Granatstein, J. H. McAdoo, and C. M. Tang, in Infrared and Millimeter Waves, edited by K. Button (Academic, 1984), Vol. 11, p. 291.

[19] K. J. Kim, in Physics of Particle Accelerators, edited by M. Month and M. Dienes, AIP Conf. Proc. No. 184 (AIP, New York, 1989), Vol. 1, pp. 184, 565.

[20] M. J. Bastiaans, J. Opt. Soc. Am. 69, 1710 (1979).

[21] A. Friedman and A. Gover, Brookhaven National Laboratory NSLS Internal Report, 1987 (unpublished).

[22] I. M. Yakover, Y. Pinhasi, and A. Gover, Nucl. Instrum. Methods Phys. Res., Sect. A 375, 260 (1996).

[23] M. K. Thumm and W. Kasparek, IEEE Trans. Plasma Sci. 30, 755 (2002).

[24] E. Jerby and A. Gover, IEEE J. Quantum Electron. 21, 1041 (1984).

[25] Y. Pinhasi, Yu. Lurie, and A. Yahalom, Nucl. Instrum. Methods Phys. Res., Sect. A 475, 147 (2001).

[26] Y. Pinhasi, Yu. Lurie, A. Yahalom, and A. Abramovich, Nucl. Instrum. Methods Phys. Res., Sect. A 483, 510 (2002).

[27] A. Abramovich, M. Canter, A. Gover, J. Sokolowski, Y. M. Yakover, Y. Pinhasi, I. Schnitzer, and J. Shiloh, Phys. Rev. Lett. 82, 5257 (1999).

[28] B. Girard, Y. Lapierre, J. M. Ortega, C. Bazin, M. Billardon, P. Ellume, M. Bergher, M. Velghe, and Y. Petroff, Phys. Rev. Lett. 53, 2405 (1984).

[29] P. J. Tallerico, Nucl. Instrum. Methods Phys. Res., Sect. A 272, 218 (1988).
[30] M. Arbel, A. Abramovich, A. L. Eichnebaum, A. Gover, H. Kleinman, Y. Pinhasi, and I. M. Yakover, Phys. Rev. Lett. 86, 2561 (2001).

[31] L. R. Elias, W. M. Fairbank, J. M. J. Madey, H. A. Schwettman, and T. I. Smith, Phys. Rev. Lett. 36, 717 (1976).

[32] M. V. Krongaus, Y. Pinhasi, M. Tecimer, and A. Gover, Nucl. Instrum. Methods Phys. Res., Sect. A 445, 28 (2000).

[33] V. I. Alexeev, E. V. Alieva, K.A. Belavintsev, E. G. Bessonov, A. V. Serov, and P. A. Cherenkov, Nucl. Instrum. Methods Phys. Res., Sect. A 282, 436 (1989).

[34] M. V. Krongauz, Y. Pinhasi, and A. Gover, "Dynamics and Control of Power Buildup in a Prebunched FEL" (unpublished).

[35] W. H. Louisell, J.F. Lam, D. A. Copelland, and W. B. Colson, Phys. Rev. A 19, 288 (1979).

[36] R. Bonifacio and L. DeSalvo Susa, Nucl. Instrum. Methods Phys. Res., Sect. A 272, 551 (1988).

[37] G. R. Neil, C. L. Bohn, S. V. Benson, G. Biallas, D. Douglas, H. F. Dylla, R. Evans, J. Fugitta, A. Grippo, J. Gubeli, R. Hill, K. Jordan, R. Li, L. Merminga, P. Piot, J. Preble, M. Shinn, T. Siggins, R. Walker, and B. Yunn, Phys. Rev. Lett. 84, 662 (2000).

[38] A. Elias et al., Phys. Rev. Lett. 57, 424 (1986).

[39] A. Abramovich, M. Canter, A. Gover, J. Sokolowski, Y. M. Yakover, Y. Pinhasi, I. Schnitzer, and J. Shiloh, Phys. Rev. Lett. 82, 5257 (1999).

[40] J. G. Neumann, P. G. O'Shea, D. Demske, W. S. Graves, B. Sheehy, H. Loos, and G. L. Carr, in Proceedings of the 24th International Free-Electron Laser Conference, Chicago, IL, 2002 [Nucl. Instrum. Methods Phys. Res., Sect. A 507, 498 (2003)].

[41] A. S. Gilmour, G. K. Farney, and B. R. Gray, IEEE Trans. Electron Devices 38, 2190 (1991).

[42] S. V. Benson, Opt. Photonics News 14, 20 (2003).

[43] F. Casagraude, R. Bonifacio, and A. Airoldi, Nucl. Instrum. Methods Phys. Res., Sect. A 304, 439 (1991).

[44] Y. Pinhasi, V. Shterngartz, and A. Gover, Phys. Rev. E 54, 6774 (1996).

[45] N. M. Kroll, P. Morton, and M. N. Rosenbluth, IEEE J. Quantum Electron. 17, 1436 (1981). 